\title{
Soft and hard interface models for bonded elements
}

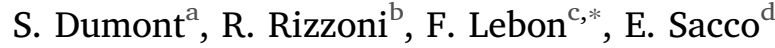 \\ ${ }^{\text {a }}$ Université de Nîmes, IMAG, CNRS UMR, 5149, Montpellier, France \\ ${ }^{\mathrm{b}} \mathrm{DE}$, Università degli Studi di Ferrara, Italy \\ ${ }^{\mathrm{c}}$ Aix-Marseille Univ, CNRS, Centrale Marseille, LMA, Marseille, France \\ d DiSt, Università degli Studi di Napoli Federico II, Italy
}

\begin{abstract}
The present paper deals with the modeling of bonded interfaces adopting the asymptotic expansion technique. The equilibrium problem of a composite body made of two adherents issn perfect contact with an elastic interface is considered and a classical rescaling technique is introduced. The asymptotic expansion method is reviewed; in fact, the representation form for the displacement and stress vector fields are introduced and tsshe effect of higher order terms is taken into account. Using the classical scheme of matched asymptotic expansions, the interface conditions are obtained. The cases of hard and soft interfaces are considered: the first is derived assuming the elasticity coefficients independent of the adherent thickness, the second considers the elasticity properties linearly depending on the thickness. Numerical investigations are performed in the framework of the finite element method. In particular, comparisons of the results obtained by modeling the adhesive as a continuum material (discretized in finite elements even in the thickness) with the results carried out using hard, soft interface models at the first and higher order expansion are performed.
\end{abstract}

\section{Introduction}

During the last decades, the interest in bonded structures, obtained by assembling different parts made of possibly different materials to compose a unique construction, is strongly increased. Bonded structures are manufactured for civil, marine and aeronautic applications. The advantages offered by the modern bonding techniques are numerous; among the others, it can be mentioned that they allow the assembly of various substrates, the assembly of materials with very different thicknesses, the connection without any deterioration of the adherents, the simplification in the design, the lightening of structures, the attenuation of vibrations, the control of the stiffness of the assemblies, the easy automation. On the other hand, these techniques could lead to limited heat resistance and short life cycle of the bonded joint; in addition, they can require some precautions of implementation because they could present resistance only to specific loading types.

As a consequence of the interest in assembled structures, the issues related to the bonding techniques, in particular their modeling and analysis represent active research fields; they concern, at least, the following subjects:

- adaptation of structures to new rules

- reinforcement of existing structures
- repair of existing structures

- realization of new and more durable structures

- development of new structural concepts.

One of the most interesting and commonly use of the bonding techniques in civil engineering constructions consists in the application of externally bonded fiber reinforced polymers (FRP) for the strengthening of existing structures. In particular, FRP are successfully adopted for the reinforcement and the repair of reinforced or prestressed concrete as well as masonry structures. They are applied on the external surfaces of concrete or masonry element by a very thin epoxy resin layer.

Many studies have been developed in the last decades concerning the safety increase related to the use of the structural strengthening with of FRP for concrete and for masonry elements, as discussed, among the other, in Refs. [2,10,11,15-18,35] and in the references therein. The objective of these studies is the evaluation of the increase of safety and lifetime of the structure, ensuring a reduced overall cost of intervention in the framework of the new ecological constraints.

A further very intriguing and challenging reinforcement technique is the application of Fiber Reinforced Cementitious Matrix (FRCM), that appears very suitable for masonry structures. FRCM consists of fibers in the form of fabric meshes, embedded into a cement-based matrix and

\footnotetext{
* Corresponding author.

E-mail address: lebon@lma.cnrs-mrs.fr (F. Lebon).
} 
directly bonded on the external surfaces of masonry support [4,19,25,26,33].

Taking into account that different adhesive materials are available in the market, the safe and advantageous use of the bonding techniques requires the development of efficient experimental procedures (i.e. mechanical, thermal, aging, chemical, non destructive testing) and robust models for the structural analysis. It could be remarked that satisfactory models for investigating the bonding effectiveness should take into account the significantly different stiffness, strength and geometrical thickness of the available adhesive materials.

A wide number of scientific works on the subject of the interface modeling can be found in literature. They are mostly based on the assumption of a simple kinematics governed by the relative displacement arising among the two surfaces defining the interface. As a consequence of the chosen kinematics, the stress state is defined at interface as the traction associated to the plane of the interface. Linear and nonlinear models are available in the literature, accounting for various inelastic effects, such damage, plasticity, unilateral contact, friction, interlocking, dilatancy, viscous behavior and so on. Interfaces are used for very different problems and also at very different scales, from the tectonic to the nano scale.

In literature, the first analytical method for the stress analysis of bonded joints was the shear-lag model developed by Volkersen [34]. This model is able to account only for the shear stresses arising along the bonding. The Volkersen's model was improved by Goland and Reissner [14], by Demarkles [7] and, later, by Erdogan and Ratwani [12], in order to include the local bending effect in the computations.

Two types of approaches can be distinguished in the interface modeling: the phenomenological and the micromechanical approaches. Phenomenological models are deduced directly from experiments. Effective nonlinear interface phenomenological models have been proposed, among the others, in Refs. [13,20,21] for the analysis of masonry walls. Raous et al. [27] presented an interface model able to couple damage and friction in the framework of consistent thermodynamics. The second type of approach for interface modeling is based on micromechanical considerations and the interface response is defined through homogenization or other techniques. A recent example of micromechanical interface model can be found in Serpieri et al. [31], where the presence and evolution of microcracks, the interlocking and dilatancy effects are accounted for considering a simplified geometry at the interface microscale. Indeed, the model is an evolution of an idea proposed in Ref. [1], where a simplified micromechanical analysis is performed and an interface model is recovered for investigating the fiber pull-in and the response of heterogeneous masonry walls. Within the same framework, an interface model whose damage is coupled with the bulk damage occurring in the adherent has been proposed in Ref. [22]. A more sophisticated micromechanical model accounting for crack closure, frictional effect and damage evolution has been presented in Ref. [30].

The asymptotic expansion techniques [5] can also be considered as belonging to the second type of approaches, i.e., they can be regarded as micromechanical models linking the interface response to the behavior of the material layer constituting the bonding. According to these techniques, the thickness and, possibly, the stiffness of the glue are considered as small parameters, vanishing in the limit theory. The glue vanishes geometrically but it remains in the equations under the form of a relation linking the stress vector to the jump in the displacement (or the rate in the displacement).

The aim of this paper is to propose mathematical and numerical methods based on asymptotic expansions for the modeling of bonded interfaces and to analyze some examples in order to show the efficiency of the methodology. In particular, the analysis is limited to the case of linear elastic response of the adhesive and adherents and the modeling is framed in the contest of small strains and small displacements theory. Even though in the context of linear elasticity, the proposed method can be very useful for design, because it allows to take into account failure occurring either within the adhesive (cohesive failure) or at the adhesive/adherent interface (interface failure).

In the first part of the paper, some recent results of asymptotic analysis for soft or stiff interfaces are reviewed [9,28,29]. First, the equilibrium problem of a composite body made of two adherents in perfect contact with an elastic interface is considered and a classical rescaling technique is introduced. The weak form of the equilibrium equations together with the matching conditions are presented. Introducing the parameter $\varepsilon$ controlling the interface thickness, two different cases are considered: the case of a hard material interface, whose elasticity coefficients are independent of $\varepsilon$, and the case of a soft material interface, whose elasticity coefficients rescale like $\varepsilon$.

For the two cases, the asymptotic expansions of the displacement and stress vector fields are introduced and the effect of higher order terms is taken into account. Using the classical scheme of matched asymptotic expansions, the interface conditions are obtained. These conditions, modeling the behavior of a very thin adhesive interface constituted of a soft or a hard material, are reviewed in Subsections 2.1 and 2.2. These interfaces equations are, in fact, relations linking the stress vector field and its jump to the displacement vector field and its jump at the interface, which is the geometric limit of the adhesive layer as its thickness parameter $\varepsilon$ goes to zero.

Following a Remark already introduced in Ref. [28], we devote Subsection 2.3 to implicit interface conditions, which take into account at the same time the interface conditions evaluated at the orders zero and one, for both the two considered cases of hard and soft interfaces. This implicit form of interface conditions takes into account higher order terms of the asymptotic expansions and it is, thus, expected to provide a better approximation of the behavior of the thin adhesive interface when compared to the classical spring-type interface law or to the case of perfect contact between the adherents. The second part of the paper is devoted to the finite element implementation and the numerical simulations. The mechanical and numerical efficiency of the proposed methodology is discussed in the conclusion section.

\section{Asymptotic analysis for soft and hard interfaces}

A thin adhesive layer $B^{\varepsilon}$ with cross-section $S$ and uniform small thickness $\varepsilon \ll \ell$ is considered, $S$ being an open bounded set in $R^{2}$ with a smooth boundary and $\ell$ a characteristic dimension of $S$. The layer $B^{\varepsilon}$ lies between two adherents bodies, occupying the reference configurations $\Omega_{ \pm}^{\varepsilon} \subset R^{3}$. $S_{ \pm}^{\varepsilon}$ is taken to denote the two surfaces between the interface and the adherents and $\Omega^{\varepsilon}=\Omega_{ \pm}^{\varepsilon} \cup S_{ \pm}^{\varepsilon} \cup B^{\varepsilon}$ is taken to denote the composite system comprising the adhesive and the two adherents. The adhesive layer $B^{\varepsilon}$ and the adherents $\Omega_{+}^{\varepsilon}, \Omega_{-}^{\varepsilon}$ are assumed to be perfectly bonded; in particular, the displacement and stress vector fields, $\mathbf{u}^{\varepsilon}$ and $\sigma^{\varepsilon} \mathbf{i}_{3}$, are assumed to be continuous across $S_{ \pm}^{\varepsilon}$, with $\mathbf{i}_{3}$ the versor of the direction perpendicular to the plane surfaces $S_{ \pm}^{\varepsilon}$.

All materials of the composite system $\Omega^{\varepsilon}$ are assumed to be homogeneous and linear elastic, with $\mathbf{a}_{ \pm}$and $\mathbf{b}^{\varepsilon}$ the elasticity tensors of the adherents and the adhesive, respectively. The tensors $\mathbf{a}_{ \pm}, \mathbf{b}^{\varepsilon}$ are assumed to be symmetric and positive definite, with the minor and major symmetries. The adherents are subjected to a body force density f: $\Omega_{ \pm}^{\varepsilon} \mapsto R^{3}$ and to a surface force density $\mathbf{g}: \Gamma_{g}^{\varepsilon} \mapsto R^{3}$ on $\Gamma_{g}^{\varepsilon} \subset\left(\partial \Omega_{+}^{\varepsilon} \backslash S_{+}^{\varepsilon}\right) \cup\left(\partial \Omega_{-}^{\varepsilon} \backslash S_{-}^{\varepsilon}\right)$. Body forces in the adhesive are negligeable. The loads are assumed to be independent of $\varepsilon$.

The asymptotic approach proposed in Refs. [9,28,29] is based on the assumption that stable equilibrium configurations of the composite body minimize its total energy:

$$
\begin{aligned}
E^{\varepsilon}(\mathbf{u})= & \int_{\Omega_{ \pm}^{\varepsilon}}\left(\frac{1}{2} \mathbf{a}_{ \pm}(\mathbf{e}(\mathbf{u})) \cdot \mathbf{e}(\mathbf{u})-\mathbf{f} \cdot \mathbf{u}\right) d V_{\mathbf{x}}-\int_{\Gamma_{g}^{\varepsilon}} \mathbf{g} \cdot \mathbf{u} d A_{\mathbf{x}} \\
& +\int_{B^{\varepsilon}} \frac{1}{2} \mathbf{b}^{\varepsilon}(\mathbf{e}(\mathbf{u})) \cdot \mathbf{e}(\mathbf{u}) d V_{\mathbf{x}},
\end{aligned}
$$

in the space of kinematically admissible displacements:

$V^{\varepsilon}=\left\{\mathbf{u} \in H\left(\Omega^{\varepsilon} ; R^{3}\right): \mathbf{u}=0\right.$ on $\left.\Gamma_{u}^{\varepsilon}\right\}$, 
where $H\left(\Omega^{\varepsilon} ; R^{3}\right)$ is the space of the vector-valued functions on the set $\Omega^{\varepsilon}$, which are continuous and differentiable as many times as necessary.

Given suitable regularity assumptions, a classical result provides the existence of a unique minimizer $\mathbf{u}^{\varepsilon}$ in $V^{\varepsilon}$ [6, Theorem6.3-2].

For the asymptotic analysis a standard change of variables, mapping the adhesive domain into a domain of unit height, is introduced [6].

After the above changes of variables, the interface occupies the domain:

$B=\left\{\left(z_{1}, z_{2}, z_{3}\right) \in R^{3}:\left(z_{1}, z_{2}\right) \in S,\left|z_{3}\right|<\frac{1}{2}\right\}$

and the adherents occupy the domains:

$\Omega_{ \pm}=\Omega_{ \pm}^{\varepsilon} \pm \frac{1}{2}(1-\varepsilon) \mathbf{i}_{3}$.

The sets $B$ and $\Omega_{ \pm}$are the rescaled configuration of the adhesive interface and of the adherents, respectively. Taken $S_{ \pm}=\left\{\left(z_{1}, z_{2}, z_{3}\right) \in R^{3}:\left(z_{1}, z_{2}\right) \in S, z_{3}= \pm \frac{1}{2}\right\}$ to denote the interfaces between $B$ and $\Omega_{ \pm}$, the set $\Omega=\Omega_{+} \cup \Omega_{-} \cup B \cup S_{+} \cup S_{-}$is the rescaled configuration of the composite system.

Using the above changes of variables and denoting by $\overline{\mathbf{f}}$ and $\overline{\mathbf{g}}$ the body force and surface force fields from the rescaled adhesive, respectively, the total energy of the rescaled configuration takes the form:

$\mathscr{E}^{\varepsilon}\left(\hat{\mathbf{u}}^{\varepsilon}, \overline{\mathbf{u}}^{\varepsilon}\right):=\int_{\Omega_{ \pm}}\left(\frac{1}{2} \mathbf{a}_{ \pm}\left(\mathbf{e}\left(\overline{\mathbf{u}}^{\varepsilon}\right)\right) \cdot \mathbf{e}\left(\overline{\mathbf{u}}^{\varepsilon}\right)-\overline{\mathbf{f}} \cdot \overline{\mathbf{u}}^{\varepsilon}\right) d V_{\mathbf{z}}-\int_{\Gamma_{g}} \overline{\mathbf{g}} \cdot \overline{\mathbf{u}}^{\varepsilon} d A_{\mathbf{z}}$

$+\int_{B} \frac{1}{2}\left(\varepsilon^{-1} \mathbf{K}_{\varepsilon}^{33}\left(\hat{\mathbf{u}}_{, 3}^{\varepsilon}\right) \cdot \hat{\mathbf{u}}_{, 3}^{\varepsilon}+2 \mathbf{K}_{\varepsilon}^{\alpha 3}\left(\hat{\mathbf{u}}_{, \alpha}^{\varepsilon}\right) \cdot \hat{\mathbf{u}}_{, 3}^{\varepsilon}+\varepsilon \mathbf{K}_{\varepsilon}^{\alpha \beta}\left(\hat{\mathbf{u}}_{, \alpha}^{\varepsilon}\right) \cdot \hat{\mathbf{u}}_{, \beta}^{\varepsilon}\right) d V_{\mathbf{z}}$,

where the matrices $\mathbf{K}_{\varepsilon}^{j l}$ (with $j, l=1,2,3$ ) are defined as:

$\left(K_{\varepsilon}^{j l}\right)_{k i}:=b_{i j k l}^{\varepsilon}$.

In view of the symmetry properties of the elasticity tensor $\mathbf{b}^{\varepsilon}$, the matrices $\mathbf{K}_{\varepsilon}^{l j}$ have the property that $\mathbf{K}_{\varepsilon}^{j l}=\left(\mathbf{K}_{\varepsilon}^{l j}\right)^{T}$, with $j, l=1,2,3$.

For an isotropic material with Lamé coefficients $\hat{\lambda}_{\varepsilon}, \hat{\mu}_{\varepsilon}$, the matrices $\mathbf{K}_{\varepsilon}^{l j}$ take the simple form

$\mathbf{K}^{l l}=\left(2 \hat{\mu}_{\varepsilon}+\hat{\lambda}_{\varepsilon}\right) \mathbf{i}_{l} \otimes \mathbf{i}_{l}+\hat{\mu}_{\varepsilon}\left(\mathbf{i}_{m} \otimes \mathbf{i}_{m}+\mathbf{i}_{n} \otimes \mathbf{i}_{n}\right), \quad m \neq l, n \neq l$,

$\mathbf{K}^{l j}=\hat{\mu}_{\varepsilon} \mathbf{i}_{l} \otimes \mathbf{i}_{j}+\hat{\lambda}_{\varepsilon} \mathbf{i}_{j} \otimes \mathbf{i}_{l}, \quad l \neq j$.

Next, the existence of asymptotic expansions of the original displacement fields $\mathbf{u}^{\varepsilon}$ and of the rescaled displacement fields $\hat{\mathbf{u}}^{\varepsilon}, \hat{\mathbf{u}}^{\varepsilon}$ with respect to the small parameter $\varepsilon$ is assumed:

$\mathbf{u}^{\varepsilon}(\mathbf{x})=\mathbf{u}^{0}(\mathbf{x})+\varepsilon \mathbf{u}^{1}(\mathbf{x})+\varepsilon^{2} \mathbf{u}^{2}(\mathbf{x})+o\left(\varepsilon^{2}\right)$,

$\hat{\mathbf{u}}^{\varepsilon}(\mathbf{z})=\hat{\mathbf{u}}^{0}(\mathbf{z})+\varepsilon \hat{\mathbf{u}}^{1}(\mathbf{z})+\varepsilon^{2} \hat{\mathbf{u}}^{2}(\mathbf{z})+o\left(\varepsilon^{2}\right)$,

$\overline{\mathbf{u}}^{\varepsilon}(\mathbf{z})=\overline{\mathbf{u}}^{0}(\mathbf{z})+\varepsilon \overline{\mathbf{u}}^{1}(\mathbf{z})+\varepsilon^{2} \overline{\mathbf{u}}^{2}(\mathbf{z})+o\left(\varepsilon^{2}\right)$.

Lastly, matching conditions are introduced based on the continuity of the traction and displacement vector fields $\sigma^{\varepsilon} \mathbf{i}_{3}, \mathbf{u}^{\varepsilon}$ at the interfaces $S_{+}^{\varepsilon}$ in the initial configuration and on the continuity of the traction and displacement vector fields $\hat{\sigma}^{\varepsilon} \mathbf{i}_{3}, \hat{\mathbf{u}}^{\varepsilon}, \bar{\sigma}^{\varepsilon} \mathbf{i}_{3}, \overline{\mathbf{u}}^{\varepsilon}$ at the interfaces $S_{ \pm}$in the rescaled configuration [28].

Using the matching conditions, any trasmission condition obtained in terms of the rescaled felds $\bar{\sigma}^{\varepsilon} \mathbf{i}_{3}, \overline{\mathbf{u}}^{\varepsilon}$ can be reformulated in terms of the stress and displacement vector fields $\sigma^{i} \mathbf{i}_{3}, \mathbf{u}^{i}, \quad i=0,1,2, \ldots$ defined on the limit configuration, which is the geometric limit of the initial configuration as the thickness of the adhesive interface $\varepsilon$ vanishes. This is possible because the matching conditions provide a link between the fields evaluated at $x_{3}=0^{ \pm}$and the rescaled fields evaluated at $z_{3}=( \pm 1 / 2)^{ \pm}$. In particular, one notes the following useful relations:

$\left[\left[\mathbf{u}^{0}\right]\right]=\left[\overline{\mathbf{u}}^{0}\right]$,

$\left[\left[\mathbf{u}^{1}\right]\right]=\left[\overline{\mathbf{u}}^{1}\right]-\ll \mathbf{u}_{, 3}^{0} \gg$,

$\left[\left[\mathbf{u}^{\varepsilon}\right]\right]=\left[\overline{\mathbf{u}}^{\varepsilon}\right]-\varepsilon \ll \mathbf{u}_{3}^{\varepsilon} \gg+o(\varepsilon)$, $\left[\left[\sigma_{i 3}^{0}\right]\right]=\left[\bar{\sigma}_{i 3}^{0}\right], \quad i=1,2,3$,

$\left[\left[\sigma_{i 3}^{1}\right]\right]=\left[\bar{\sigma}_{i 3}^{1}\right]-\ll \sigma_{i 3,3}^{0} \gg, \quad i=1,2,3$,

$\left[\left[\sigma_{i 3}^{\varepsilon}\right]\right]=\left[\bar{\sigma}_{i 3}^{\varepsilon}\right]-\varepsilon \ll \sigma_{i 3,3}^{\varepsilon} \gg+o(\varepsilon), \quad i=1,2,3$,

$\ll \mathbf{u}^{0} \gg=\left\langle\overline{\mathbf{u}}^{0}\right\rangle$,

$\ll \mathbf{u}^{1} \gg=<\overline{\mathbf{u}}^{1}>-\frac{1}{4}\left[\left[\mathbf{u}_{, 3}^{0}\right]\right]$,

$\ll \mathbf{u}^{\varepsilon} \gg=\left\langle\overline{\mathbf{u}}^{\varepsilon}>-\frac{\varepsilon}{4}\left[\left[\mathbf{u}_{, 3}^{\varepsilon}\right]\right]+o(\varepsilon)\right.$,

$\ll \sigma_{i 3}^{0} \gg=\left\langle\bar{\sigma}_{i 3}^{0}\right\rangle, i=1,2,3$,

$\ll \sigma_{i 3}^{1} \gg=<\sigma_{i 3}^{1}>-\frac{1}{4}\left[\left[\sigma_{i 3,3}^{0}\right]\right], \quad i=1,2,3$,

$\ll \sigma_{i 3}^{\varepsilon} \gg=\left\langle\bar{\sigma}_{i 3}^{\varepsilon}>-\frac{\varepsilon}{4}\left[\left[\sigma_{i 3,3}^{\varepsilon}\right]\right]+o(\varepsilon), \quad i=1,2,3\right.$,

where the notations

$[f(\mathbf{z})]:=\left(f\left(\mathbf{z},(+1 / 2)^{+}\right)-f\left(\mathbf{z},(-1 / 2)^{-}\right)\right), \quad \mathbf{z} \in S$,

$<f(\mathbf{x})>:=\frac{1}{2}\left(f\left(\overline{\mathbf{z}},(+1 / 2)^{+}\right)+f\left(\overline{\mathbf{z}},(-1 / 2)^{-}\right)\right), \quad \mathbf{z} \in S$,

$[[f(\mathbf{x})]]:=\left(f\left(\mathbf{x}, 0^{+}\right)-f\left(\mathbf{x}, 0^{-}\right)\right), \quad \mathbf{x} \in S$,

$\ll f(\mathbf{x}) \gg:=\frac{1}{2}\left(f\left(\overline{\mathbf{x}}, 0^{+}\right)+f\left(\overline{\mathbf{x}}, 0^{-}\right)\right), \quad \mathbf{x} \in S$.

have been introduced.

In the next subsections, two different cases of material behavior for the interface can considered. In the first case, the interface is considered hard, meaning that the elasticity coefficients $b_{i j k l}^{\varepsilon}$ are assumed to be independent of $\varepsilon$; in the second case, the interface is considered soft, meaning that the elasticity coefficients $b_{i j k l}^{\varepsilon}$ are assumed to linearly rescale with the thickness $\varepsilon$.

\subsection{Adhesive made of a hard material}

The elasticity coefficients $b_{i j k l}^{\varepsilon}$ of an adhesive made of a hard material are assumed to be independent of $\varepsilon$ :

$b_{i j k l}^{\varepsilon}=b_{i j k l}$.

Accordingly, $\mathbf{K}^{j l}$ are taken to denote the matrices such that $\left(\mathbf{K}^{j l}\right)_{k i}:=b_{i j k l}$.

In Ref. [28], it is shown that the transmission conditions obtained for the hard interface in the rescaled configuration are

$\left[\bar{\sigma}^{0} \mathbf{i}_{3}\right]=0$,

$\left[\overline{\mathbf{u}}^{0}\right]=0$,

$\left[\bar{\sigma}^{1} \mathbf{i}_{3}\right]=-\mathbf{K}^{3 \alpha}\left(\mathbf{K}^{33}\right)^{-1}\left(\bar{\sigma}_{, \alpha}^{0} \mathbf{i}_{3}\right)-\left(\mathbf{K}^{\alpha \beta}+\mathbf{K}^{3 \alpha}\left(\mathbf{K}^{33}\right)^{-1} \mathbf{K}^{\beta 3}\right) \overline{\mathbf{u}}_{, \alpha \beta}^{0}$,

$\left[\overline{\mathbf{u}}^{1}\right]=\left(\mathbf{K}^{33}\right)^{-1}\left(\bar{\sigma}^{0} \mathbf{i}_{3}-\mathbf{K}^{\alpha 3} \overline{\mathbf{u}}_{, \alpha}^{0}\right)$.

The matching conditions (12), (13), (15), (16) allow to transform the above transmission conditions into interface conditions appropriate for a hard interface:

$\left[\left[\sigma^{0} \mathbf{i}_{3}\right]\right]=0$,

$\left[\left[\mathbf{u}^{0}\right]\right]=0$,

$\left[\left[\sigma^{1} \mathbf{i}_{3}\right]\right]=-\mathbf{K}^{3 \alpha}\left(\mathbf{K}^{33}\right)^{-1}\left(\sigma_{, \alpha}^{0} \mathbf{i}_{3}\right)-\left(\mathbf{K}^{\alpha \beta}-\mathbf{K}^{3 \alpha}\left(\mathbf{K}^{33}\right)^{-1} \mathbf{K}^{\beta 3}\right) \mathbf{u}_{, \alpha \beta}^{0}$

$-\ll \sigma_{, 3}^{0} \mathbf{i}_{3} \gg$,

$\left[\left[\mathbf{u}^{1}\right]\right]=\left(\mathbf{K}^{33}\right)^{-1}\left(\sigma^{0} \mathbf{i}_{3}-\mathbf{K}^{\alpha 3} \mathbf{u}_{, \alpha}^{0}\right)-\ll \mathbf{u}_{3}^{0} \gg$.

In the particular case of an interface made of an isotropic material, relations (7) and (8) can be applied to specialize the interface laws 
(33)-(36) as follows:

$$
\begin{aligned}
{\left[\left[\sigma_{i 3}^{0}\right]\right]=} & 0, \quad i=1,2,3, \\
{\left[\left[u_{i}^{0}\right]\right]=} & 0, \quad i=1,2,3, \\
{\left[\left[\sigma_{13}^{1}\right]\right]=} & -4 \hat{\mu}(\hat{\mu}+\hat{\lambda})(2 \hat{\mu}+\hat{\lambda})^{-1} u_{1,11}^{0}-\hat{\mu} u_{1,22}^{0} \\
& -\hat{\mu}(2 \hat{\mu}+3 \hat{\lambda})(2 \hat{\mu}+\hat{\lambda})^{-1} u_{2,21}^{0} \\
& -\hat{\lambda}(\hat{\mu}+\hat{\lambda})^{-1} \sigma_{33,1}^{0}-\ll \sigma_{13,3}^{0} \gg, \\
{\left[\left[\sigma_{23}^{1}\right]\right]=} & -4 \hat{\mu}(\hat{\mu}+\hat{\lambda})(2 \hat{\mu}+\hat{\lambda})^{-1} u_{2,22}^{0}-\hat{\mu} u_{2,11}^{0} \\
& -\hat{\mu}(2 \hat{\mu}+3 \hat{\lambda})(2 \hat{\mu}+\hat{\lambda})^{-1} u_{1,22}^{0} \\
& -\hat{\lambda}(\hat{\mu}+\hat{\lambda})^{-1} \sigma_{33,2}^{0}-\ll \sigma_{23,3}^{0} \gg, \\
{\left[\left[\sigma_{33}^{1}\right]\right]=} & -\sigma_{13,1}^{0}-\sigma_{23,2}^{0}-\ll \sigma_{33,3}^{0} \gg, \\
{\left[\left[u_{\alpha}^{1}\right]\right]=} & \hat{\mu}^{-1} \sigma_{\alpha 3}^{0}-u_{3, \alpha}^{0}-\ll u_{\alpha, 3}^{0} \gg, \quad \alpha=1,2, \\
{\left[\left[u_{3}^{1}\right]\right]=} & (2 \hat{\mu}+\hat{\lambda})^{-1}\left(\sigma_{33}^{0}-\hat{\lambda}\left(u_{1,1}^{0}+u_{2,2}^{0}\right)\right)-\ll u_{3,3}^{0} \gg,
\end{aligned}
$$

where $\hat{\mu}=\hat{\mu}_{\varepsilon}$ and $\hat{\lambda}=\hat{\lambda}_{\varepsilon}$ are the Lamé's coefficients of the adhesive, independent of $\varepsilon$.

\subsection{Adhesive made of a soft material}

An adhesive soft material is defined to be such that its elasticity coefficients $b_{i j k l}^{\varepsilon}$ rescale linearly with $\varepsilon$ :

$b_{i j k l}^{\varepsilon}=\varepsilon b_{i j k l}$.

Accordingly, $\mathbf{K}^{j l}$ are still taken to denote the matrices such that $\left(K^{j l}\right)_{k i}:=b_{i j k l}$, with $b_{i j k l}$ as in (39).

In Ref. [28], it has been shown that for the soft interface in the rescaled configuration the following trasmission conditions can be obtained:

$\left[\bar{\sigma}^{0} \mathbf{i}_{3}\right]=0$,

$\left[\overline{\mathbf{u}}^{0}\right]=\left(\mathbf{K}^{33}\right)^{-1}\left(\bar{\sigma}^{0} \mathbf{i}_{3}\right)$,

$\left[\bar{\sigma}^{1} \mathbf{i}_{3}\right]=-\mathbf{K}^{3 \alpha}\left(\mathbf{K}^{33}\right)^{-1}\left(\bar{\sigma}_{, \alpha}^{0} \mathbf{i}_{3}\right)$,

$\left[\overline{\mathbf{u}}^{1}\right]=\left(\mathbf{K}^{33}\right)^{-1}\left(<\bar{\sigma}^{1} \mathbf{i}_{3}>-\mathbf{K}^{\alpha 3}<\overline{\mathbf{u}}_{, \alpha}^{0}>\right)$.

Using the matching conditions (12), (13), (15), (16), the following interface laws appropriate for a soft interface are obtained:

$\left[\left[\sigma^{0} \mathbf{i}_{3}\right]\right]=0$,

$\left[\left[\mathbf{u}^{0}\right]\right]=\left(\mathbf{K}^{33}\right)^{-1}\left(\sigma^{0} \mathbf{i}_{3}\right)$,

$\left[\left[\sigma^{1} \mathbf{i}_{3}\right]\right]=-\mathbf{K}^{3 \alpha}\left(\mathbf{K}^{33}\right)^{-1}\left(\sigma_{, \alpha}^{0} \mathbf{i}_{3}\right)-\ll \sigma_{, 3}^{0} \mathbf{i}_{3} \gg$,

$\left[\left[\mathbf{u}^{1}\right]\right]=\left(\mathbf{K}^{33}\right)^{-1}\left(\ll \sigma^{1} \mathbf{i}_{3} \gg+\frac{1}{4}\left[\left[\sigma_{, 3}^{0} \mathbf{i}_{3}\right]\right]-\mathbf{K}^{\alpha 3} \ll \mathbf{u}_{, \alpha}^{0} \gg\right)-\ll \mathbf{u}_{, 3}^{0} \gg$.

In case of a soft isotropic interface, the above relations specialize as

$\left[\left[\sigma_{i 3}^{0}\right]\right]=0, \quad i=1,2,3$,

$\left[\left[u_{\alpha}^{0}\right]\right]=(\hat{\mu})^{-1} \sigma_{\alpha 3}^{0}, \quad \alpha=1,2$,

$\left[\left[u_{3}^{0}\right]\right]=(2 \hat{\mu}+\hat{\lambda})^{-1} \sigma_{33}^{0}$,

$\left[\left[\sigma_{\alpha 3}^{1}\right]\right]=-\hat{\lambda}(2 \hat{\mu}+\hat{\lambda})^{-1} \sigma_{33, \alpha}^{0}-\ll \sigma_{\alpha 3,3}^{0} \gg, \quad \alpha=1,2$,

$\left[\left[\sigma_{33}^{1}\right]\right]=-\sigma_{13,1}^{0}-\sigma_{23,2}^{0}-\ll \sigma_{33,3}^{0} \gg$,

$\left[\left[u_{\alpha}^{1}\right]\right]=\hat{\mu}^{-1}\left(\ll \sigma_{\alpha 3}^{1} \gg+\frac{1}{4}\left[\left[\sigma_{\alpha 3,3}^{0}\right]\right]\right)-u_{3, \alpha}^{0}-\ll u_{\alpha, 3}^{0} \gg, \quad \alpha=1,2$,

$\left[\left[u_{3}^{1}\right]\right]=(2 \hat{\mu}+\hat{\lambda})^{-1}\left(\ll \sigma_{33}^{1} \gg+\frac{1}{4}\left[\left[\sigma_{33,3}^{0}\right]\right]-\hat{\lambda}\left(u_{1,1}^{0}+u_{2,2}^{0}\right)\right)-\ll u_{3,3}^{0} \gg$.

with $\hat{\mu}_{\varepsilon}=\hat{\mu} \varepsilon$ and $\hat{\lambda}_{\varepsilon}=\hat{\lambda} \varepsilon$.

\subsection{Implicit form of the transmission conditions}

In Ref. [28], it has been shown that it is possible to obtain a condensed form of transmission conditions summarizing both the orders zero and one of the two cases of soft and hard interface materials in only one couple of equations. The result is the following implicit formulation of transmission conditions for the rescaled interface:

$\left[\bar{\sigma}^{\varepsilon} \mathbf{i}_{3}\right]=-\varepsilon\left(\mathbf{K}_{\varepsilon}^{3 \alpha}\left(\mathbf{K}_{\varepsilon}^{33}\right)^{-1}<\bar{\sigma}_{, \alpha}^{\varepsilon} \mathbf{i}_{3}>+\left(\mathbf{K}_{\varepsilon}^{\alpha \beta}-\mathbf{K}_{\varepsilon}^{3 \alpha}\left(\mathbf{K}_{\varepsilon}^{33}\right)^{-1} \mathbf{K}_{\varepsilon}^{\beta 3}\right)<\overline{\mathbf{u}}_{, \alpha \beta}^{\varepsilon}>\right)$,

$\left[\overline{\mathbf{u}}^{\varepsilon}\right]=\varepsilon\left(\mathbf{K}_{\varepsilon}^{33}\right)^{-1}\left(<\bar{\sigma}^{\varepsilon} \mathbf{i}_{3}>-\mathbf{K}_{\varepsilon}^{\alpha 3}<\overline{\mathbf{u}}_{, \alpha}^{\varepsilon}>\right)$.

These latter equations are clearly implied by (29)-(32). Indeed, they can be directly obtained by substituting (30) ((29)) summed with (32) ((31)) multiplied for $\varepsilon$ into the asymptotic expansions for the displacement (traction) field. Angle bracket parentheses have been introduced in (50) and (51) where necessary, i.e. to take into account that $\bar{\sigma}^{\varepsilon}$ and $\overline{\mathbf{u}}^{\varepsilon}$ suffer jumps.

To see that (50) and (51) imply (40)-(43), it is sufficient to substitute inside (50) and (51) the asymptotic expansions of the displacement and the traction fields together with the rescaling (39) of the elasticity coefficients adopted for the case of soft material, neglect the second order terms in $\varepsilon$ and consider the term at the orders 0 and 1 in $\varepsilon$.

Notably, the transmission conditions (50) and (51) can be transformed into interface equations, by making use of the relations (14), (17) and of the following newly derived ones:

$\ll \mathbf{u}_{, \alpha}^{\varepsilon} \gg=<\overline{\mathbf{u}}_{, \alpha}^{\varepsilon}>-\frac{\varepsilon}{4}\left[\left[\mathbf{u}_{, \alpha 3}^{\varepsilon}\right]\right]+o(\varepsilon)$,

$\ll \mathbf{u}_{, \alpha \beta}^{\varepsilon} \gg=<\overline{\mathbf{u}}_{, \alpha \beta}^{\varepsilon}>-\frac{\varepsilon}{4}\left[\left[\mathbf{u}_{, \alpha \beta 3}^{\varepsilon}\right]\right]+o(\varepsilon)$,

$\ll \sigma_{, \alpha}^{\varepsilon} \mathbf{i}_{3} \gg=\left\langle\bar{\sigma}_{, \alpha}^{\varepsilon} \mathbf{i}_{3}>-\frac{\varepsilon}{4}\left[\left[\sigma_{, \alpha 3}^{\varepsilon} \mathbf{i}_{3}\right]\right]+o(\varepsilon)\right.$.

The new interface conditions equivalent to (50) and (51) are, up to second and higher order terms in $\varepsilon$,

$\left[\left[\sigma^{\varepsilon} \mathbf{i}_{3}\right]\right]=-\varepsilon\left(\mathbf{K}_{\varepsilon}^{3 \alpha}\left(\mathbf{K}_{\varepsilon}^{33}\right)^{-1}<\left\langle\sigma_{, \alpha}^{\varepsilon} \mathbf{i}_{3}>>\left(\mathbf{K}_{\varepsilon}^{\alpha \beta}-\mathbf{K}_{\varepsilon}^{3 \alpha}\left(\mathbf{K}_{\varepsilon}^{33}\right)^{-1} \mathbf{K}_{\varepsilon}^{\beta 3}\right) \ll \mathbf{u}_{, \alpha \beta}^{\varepsilon} \gg\right)\right.$

$-\varepsilon \ll \sigma_{, 3}^{\varepsilon} \mathbf{i}_{3} \gg+o(\varepsilon)$,

$\left[\left[\mathbf{u}^{\varepsilon}\right]\right]=\varepsilon\left(\mathbf{K}_{\varepsilon}^{33}\right)^{-1}\left(\ll \sigma^{\varepsilon} \mathbf{i}_{3} \gg+\frac{\varepsilon}{4}\left[\left[\sigma_{, 3}^{\varepsilon} \mathbf{i}_{3}\right]\right]-\mathbf{K}_{\varepsilon}^{\alpha 3} \ll \mathbf{u}_{, \alpha}^{\varepsilon} \gg-\ll \mathbf{u}_{, 3}^{\varepsilon} \gg\right)$ $+o(\varepsilon)$.

Substituting $(7,8)$ into the implicit interfacial equations (55) and (56), the latter ones specialize as

$$
\begin{aligned}
{\left[\left[\sigma_{13}^{\varepsilon}\right]\right]=} & -4 \mu_{\varepsilon}\left(\mu_{\varepsilon}+\lambda_{\varepsilon}\right)\left(2 \mu_{\varepsilon}+\lambda_{\varepsilon}\right)^{-1} \ll u_{1,11}^{\varepsilon} \gg-\mu_{\varepsilon} \ll u_{1,22}^{\varepsilon} \gg \\
& -\mu_{\varepsilon}\left(2 \mu_{\varepsilon}+3 \lambda_{\varepsilon}\right)\left(2 \mu_{\varepsilon}+\lambda_{\varepsilon}\right)^{-1} \ll u_{2,21}^{\varepsilon} \gg \\
& -\lambda_{\varepsilon}\left(\mu_{\varepsilon}+\lambda_{\varepsilon}\right)^{-1} \ll \sigma_{33,1}^{\varepsilon} \gg-\ll \sigma_{13,3}^{\varepsilon} \gg \\
{\left[\left[\sigma_{23}^{\varepsilon}\right]\right]=} & -4 \mu_{\varepsilon}\left(\mu_{\varepsilon}+\lambda_{\varepsilon}\right)\left(2 \mu_{\varepsilon}+\lambda_{\varepsilon}\right)^{-1} \ll u_{2,22}^{\varepsilon} \gg-\mu_{\varepsilon} \ll u_{2,11}^{\varepsilon} \gg \\
& -\mu_{\varepsilon}\left(2 \mu_{\varepsilon}+3 \lambda_{\varepsilon}\right)\left(2 \mu_{\varepsilon}+\lambda_{\varepsilon}\right)^{-1} \ll u_{1,22}^{\varepsilon} \gg \\
& -\lambda_{\varepsilon}\left(\mu_{\varepsilon}+\lambda_{\varepsilon}\right)^{-1} \ll \sigma_{33,2}^{\varepsilon} \gg-\ll \sigma_{23,3}^{\varepsilon} \gg, \\
{\left[\left[\sigma_{33}^{\varepsilon}\right]\right]=} & -\ll \sigma_{13,1}^{\varepsilon} \gg-\ll \sigma_{23,2}^{\varepsilon} \gg-\ll \sigma_{33,3}^{\varepsilon} \gg, \\
{\left[\left[u_{\alpha}^{\varepsilon}\right]\right]=} & \varepsilon \mu_{\varepsilon}^{-1}\left(\ll \sigma_{\alpha 3}^{\varepsilon} \gg+\frac{\varepsilon}{4}\left[\left[\sigma_{\alpha 3,3}^{\varepsilon}\right]\right]\right)-\varepsilon \ll u_{3, \alpha}^{\varepsilon}+u_{\alpha, 3}^{\varepsilon} \gg, \quad \alpha=1,2
\end{aligned}
$$




$$
\begin{aligned}
{\left[\left[u_{3}^{\varepsilon}\right]\right]=} & \varepsilon\left(2 \mu_{\varepsilon}+\lambda_{\varepsilon}\right)^{-1}\left(\ll \sigma_{33}^{\varepsilon} \gg+\frac{\varepsilon}{4}\left[\left[\sigma_{33,3}^{\varepsilon}\right]\right]-\lambda_{\varepsilon} \ll u_{1,1}^{\varepsilon}+u_{2,2}^{\varepsilon} \gg\right) \\
& -\varepsilon \ll u_{3,3}^{\varepsilon} \gg .
\end{aligned}
$$

As discussed in Ref. [28], the implicit interfacial equations establishes a formal equivalence between the soft interface law and the hard interface law (cf. also $[28,5.5]$ ). These equations can thus be viewed as the interface laws for a thin isotropic interface condensing in an unique form the two cases of a soft and a hard interface. As already remarked in the Introduction, equations (56) and (55) take into account higher order terms of the asymptotic expansions and they are thus expected to provide a better approximation of the behavior of the thin adhesive interface when compared to the classical spring-type interface law or to the case of perfect contact between the adherents. This issue is explored in the next Sections by means of several numerical examples.

\section{Finite element implementation and numerical tests}

\subsection{Soft at order 0 and 1}

First, let us notice that the jump conditions for soft interfaces (40)-(43) both at order 0 and 1 can be written

$[\mathbf{u}]=\mathbf{C}<\sigma \mathbf{i}_{3}>+\mathbf{D}^{0}$

$$
\left[\sigma \mathbf{i}_{3}\right]=\mathbf{S}^{0}
$$

where we have omitted the bar sign for simplicity of notation and where

$\mathbf{C}=\left(\begin{array}{ccc}\hat{\mu} & 0 & 0 \\ 0 & \hat{\mu} & 0 \\ 0 & 0 & 2 \hat{\lambda}+\hat{\mu}\end{array}\right)$

and

$\begin{cases}S_{\alpha}^{0}=S_{3}^{0}=0, & \alpha=1,2, \\ D_{\alpha}^{0}=D_{3}^{0}=0 & \alpha=1,2,\end{cases}$

at order 0 , while

$$
\left\{\begin{array}{c}
S_{\alpha}^{0}=-\hat{\lambda}(2 \hat{\mu}+\hat{\lambda})^{-1}<\sigma_{33, \alpha}^{0}>-<\sigma_{\alpha 3,3}^{0}>, \quad \alpha=1,2, \\
S_{3}^{0}=-\sigma_{13,1}^{0}-\sigma_{23,2}^{0}-<\sigma_{33,3}^{0}> \\
D_{\alpha}^{0}=\frac{1}{4}\left[\sigma_{\alpha 3,3}^{0}\right]-<u_{3, \alpha}^{0}>-<u_{\alpha, 3}^{0}>, \quad \alpha=1,2, \\
D_{3}^{0}=\frac{1}{4}\left[\sigma_{33,3}^{0}\right]-\hat{\lambda}\left(\left[u_{1,1}^{0}\right]+\left[u_{2,2}^{0}\right]\right)-<u_{3,3}^{0}>
\end{array}\right.
$$

at order 1 .

Then, in order to write the variational form of the mechanical problem, one write the variational form of the equilibrium problem on each sub-domain $\Omega^{-}$and $\Omega^{+}$. The sum of the two equations is

$$
\begin{aligned}
& \int_{\Omega_{ \pm}} \mathbf{a}_{ \pm}(\mathbf{e}(\mathbf{u})) \cdot \mathbf{e}(\mathbf{v}) d V_{\mathbf{z}}-\int_{\Gamma} \sigma\left(\mathbf{z}, 0^{+}\right) \mathbf{n}\left(\mathbf{z}, 0^{+}\right) d A_{\mathbf{z}}-\int_{\Gamma} \sigma\left(\mathbf{z}, 0^{-}\right) \mathbf{n}\left(\mathbf{z}, 0^{-}\right) d A_{\mathbf{z}} \\
& =\int_{\Omega_{ \pm}} \mathbf{f} \cdot \mathbf{u} d V_{\mathbf{z}}+\int_{\Gamma_{g}} \mathbf{g} \cdot \mathbf{u} d A_{\mathbf{z}}
\end{aligned}
$$

which can be written

$\int_{\Omega_{ \pm}} \mathbf{a}_{ \pm}(\mathbf{e}(\mathbf{u})) \cdot \mathbf{e}(\mathbf{v}) d V_{\mathbf{z}}+\int_{\Gamma}[(\sigma \mathbf{n}) \cdot \mathbf{v}] d A_{\mathbf{z}}=\ell(\mathbf{v})$

choosing $\mathbf{n}(\mathbf{z}, 0)=\mathbf{n}\left(\mathbf{z}, 0^{-}\right)=-\mathbf{n}\left(\mathbf{z}, 0^{+}\right), \ell(\mathbf{v})=\int_{\Omega_{ \pm}} \mathbf{f} \cdot \mathbf{u} d V_{\mathbf{z}}+\int_{\Gamma_{g}} \mathbf{g} \cdot \mathbf{u} d A_{\mathbf{z}}$.

Then, using the property $[a b]=\langle a\rangle[b]+[a]\langle b\rangle$, and using (61), we obtain

$$
\begin{gathered}
\int_{\Omega_{ \pm}} \mathbf{a}_{ \pm}(\mathbf{e}(\mathbf{u})) \cdot \mathbf{e}(\mathbf{v}) d V_{\mathbf{z}}+\int_{\Gamma}\left(\mathbf{C}^{-1}[\mathbf{u}]\right) \cdot[\mathbf{v}] d A_{\mathbf{z}}=\ell(v)+\int_{\Gamma}\left(\mathbf{C}^{-1} \mathbf{D}^{0}\right) \\
\cdot[\mathbf{v}] d A_{\mathbf{z}}-\int_{\Gamma} \mathbf{S}^{0}<\mathbf{v}>d A_{\mathbf{z}} .
\end{gathered}
$$

Finally, a standard finite element method is used to solved this equation. In order to take into account the jumps in the displacements across the interface, a "flat" finite element is considered on the interface $\Gamma$ that has all nodes on $\Gamma$, the first ones related to $\Gamma_{-}$, and the other ones related to $\Omega_{+}$. It is then possible to write a stiffness matrix of this problem that is invertible and with standard error estimates (for more details, see for example [23]).

Remark. It is also possible to treat hard interface law at order one with this methods. After the computation at order 0 , the term $\mathbf{u}^{\varepsilon}=\mathbf{u}^{0}+\varepsilon \mathbf{u}^{1}$ that satisfy the jumps conditions can be computed using (37) with the following approximation that is equal to the previous one up to order 1

$$
\begin{aligned}
{\left[\left[u_{\alpha}^{\varepsilon}\right]\right]=} & \hat{\mu}^{-1} \sigma_{\alpha 3}^{\varepsilon}-u_{3, \alpha}^{0}-\ll u_{\alpha, 3}^{0} \gg=\hat{\mu}^{-1} \sigma_{\alpha 3}^{\varepsilon}+D_{\alpha}^{0}, \quad \alpha=1,2, \\
{\left[\left[u_{3}^{\varepsilon}\right]\right]=} & (2 \hat{\mu}+\hat{\lambda})^{-1}\left(\sigma_{33}^{\varepsilon}-\hat{\lambda}\left(u_{1,1}^{0}+u_{2,2}^{0}\right)\right)-\ll u_{3,3}^{0} \gg=(2 \hat{\mu}+\hat{\lambda})^{-1} \sigma_{33}^{\varepsilon}+D_{3}^{0}, \\
{\left[\left[\sigma_{13}^{\varepsilon}\right]\right]=} & -4 \hat{\mu}(\hat{\mu}+\hat{\lambda})(2 \hat{\mu}+\hat{\lambda})^{-1} u_{1,11}^{0}-\hat{\mu} u_{1,22}^{0}-\hat{\mu}(2 \hat{\mu}+3 \hat{\lambda})(2 \hat{\mu}+\hat{\lambda})^{-1} u_{2,21}^{0} \\
& -\hat{\lambda}(\hat{\mu}+\hat{\lambda})^{-1} \sigma_{33,1}^{0}-\ll \sigma_{13,3}^{0} \gg=S_{13}^{0}, \\
{\left[\left[\sigma_{23}^{\varepsilon}\right]\right]=} & -4 \hat{\mu}(\hat{\mu}+\hat{\lambda})(2 \hat{\mu}+\hat{\lambda})^{-1} u_{2,22}^{0}-\hat{\mu} u_{2,11}^{0}-\hat{\mu}(2 \hat{\mu}+3 \hat{\lambda})(2 \hat{\mu}+\hat{\lambda})^{-1} u_{1,22}^{0} \\
& -\hat{\lambda}(\hat{\mu}+\hat{\lambda})^{-1} \sigma_{33,2}^{0}-\ll \sigma_{23,3}^{0} \gg=S_{23}^{0}, \\
{\left[\left[\sigma_{33}^{\varepsilon}\right]\right]=} & -\sigma_{13,1}^{0}-\sigma_{23,2}^{0}-\ll \sigma_{33,3}^{0} \gg=S_{33}^{0} .
\end{aligned}
$$

The finite element method applied to this formulation will be called the semi-implicit formulation hereinafter, since the constraints in the jumps in displacements are treated implicitly.

\subsection{Hard at order 0 and 1}

The hard interface laws in equations (29-32) both at order 0 and 1 can be written in a general form

$[\mathbf{u}]=\mathbf{D}^{0}$

$\left[\sigma \mathbf{i}_{3}\right]=\mathbf{S}^{0}$

Unfortunately, it is no longer possible to write a standard variational formulation of the problem. However, a discontinuous Galerkin method can be used to solve the problem (see Ref. [9]).

More precisely, going back to equation (63) and using (65), we have

$$
\begin{gathered}
\int_{\Omega_{ \pm}} \mathbf{a}_{ \pm}(\mathbf{e}(\mathbf{u})) \cdot \mathbf{e}(\mathbf{v}) d V_{\mathbf{z}}+\int_{\Gamma}\left(<\mathbf{a}_{ \pm}(\mathbf{e}(\mathbf{u})) \mathbf{n}>\cdot[\mathbf{v}]+[\mathbf{u}] \cdot<\mathbf{a}_{ \pm}(\mathbf{e}(\mathbf{u})) \mathbf{n}>\right) d A_{\mathbf{z}} \\
=\ell(\mathbf{v})-\int_{\Gamma} \mathbf{S}^{0}<\mathbf{v}>d A_{\mathbf{z}} \cdot+\int_{\Gamma} \mathbf{D}^{0} \cdot<\mathbf{a}_{ \pm} \mathbf{e}(\mathbf{v}) \mathbf{n}>d A_{\mathbf{z}} .
\end{gathered}
$$

This formulation, known as the Nitsche's method [24] is unstable and the discrete operator can be non invertible after a discretization. It is then necessary to add a stabilization term such as $\frac{\beta}{h} \int_{\Gamma}[\mathbf{u}] \cdot[\mathbf{v}] d A_{\mathbf{z}}=\frac{\beta}{h} \int_{\Gamma} \mathbf{D}^{0} \cdot[\mathbf{v}] d A_{\mathbf{z}}$, where $h$ is the size of the smallest element of the finite element discretization of $\Omega_{ \pm}$considered and $\beta>0$ is a fixed number that must be sufficiently large to ensure the stability of the method. It can be shown that this formulation is equivalent to the initial problem in the sense that solutions of (66) are weak solutions of the initial problem (see Refs. [3,8,32] for the complete study of this method and for a priori and a posteriori error estimates in the case $\mathbf{D}^{0}=0$ ).

Unfortunately, this method does not work properly to solve problem (66) as soon as $\mathbf{D}^{0} \neq 0$. To overcome this difficulty, the initial problem with the two jump conditions is split into two parts, the first one to treat the jump in the displacements, the second one for the jump in the constraints.

More precisely, the unknown displacement $\mathbf{u}^{ \pm}$is written $\mathbf{u}^{ \pm}=\mathbf{z}^{ \pm}+\mathbf{w}^{ \pm}$where $\mathbf{z}^{ \pm}$and $\mathbf{w}^{ \pm}$solve the problems 


$$
\left\{\begin{array} { c c c } 
{ \operatorname { d i v } \sigma ( \mathbf { z } ^ { \pm } ) = 0 } & { \text { in } \Omega _ { \pm } } \\
{ \sigma ( \mathbf { z } ^ { \pm } ) \mathbf { n } = 0 } & { \text { on } \Gamma _ { u } } \\
{ \mathbf { z } ^ { \pm } = 0 } & { \text { on } \Gamma _ { g } } \\
{ \sigma ( \mathbf { z } ^ { \pm } ) = \mathbf { a } _ { \pm } \mathbf { e } ( \mathbf { z } ^ { \pm } ) } & { \text { in } \Omega _ { \pm } } \\
{ \mathbf { z } ^ { \pm } = \pm \frac { 1 } { 2 } \mathbf { D } ^ { 0 } } & { \text { on } \Gamma }
\end{array} \quad \left\{\begin{array}{ccc}
\operatorname{div} \sigma\left(\mathbf{w}^{ \pm}\right)=0 & \text { in } \Omega_{ \pm} \\
\sigma\left(\mathbf{w}^{ \pm}\right) \mathbf{n}=0 & \text { on } \Gamma_{u} \\
\mathbf{w}^{ \pm}=0 & \text { on } \Gamma_{0} g \\
\sigma\left(\mathbf{w}^{ \pm}\right)=\mathbf{a}_{ \pm} \mathbf{e}\left(\mathbf{w}^{ \pm}\right) & \text {in } \Omega_{ \pm} \\
{[\mathbf{w}]=0} & \text { on } \Gamma \\
{[\sigma(\mathbf{w}) \mathbf{n}]=\mathbf{S}^{0}-[\sigma(\mathbf{z}) \mathbf{n}]} & \text { on } \Gamma
\end{array}\right.\right.
$$

since $[\mathbf{w}]=\mathbf{w}^{+}-\mathbf{w}^{-}=[\mathbf{u}]-\mathbf{z}^{+}+\mathbf{z}^{-}=\left(1-\frac{1}{2}-\frac{1}{2}\right) \mathbf{D}^{0}=0$. The two first problems in the left, defined in both $\Omega_{+}$and $\Omega_{-}$, are standard and can be solved simultaneously using a standard finite element method. The problem on the right of (67) is solved using the Nitsche's method developed above.

\section{Numerical study}

\subsection{Bond of two square bodies}

Aim of the first study is to derive the different responses obtained adopting the soft and hard interface models, described in the previous sections, comparing that solutions with a reference solution evaluated performing a finite element analysis, which considers adherents and adhesive as solid continua.

A structural system obtained assembling two adherents by means of an adhesive interface, as schematically illustrated in Fig. 1, is considered. Each adherent is approximatively a square with dimensions $5 \times(5-\varepsilon / 2)$, while the adhesive thickness is $\varepsilon$, so that the total height of the structural system is $L=10$, where all lengths are measured in meters. The structural scheme is subjected to a uniform vertical load on the top edge $F=2 \mathrm{MPa}$, and to a horizontal load $P=1 \mathrm{MPa}$ distributed on a length equal to 1 above the top.

The materials constituting the adherents and the adhesive are assumed to be isotropic; in particular, the Young's modulus of the adherents is set equal to $E_{a}=100 \mathrm{GPa}$, while the Poissons's ratio of all components is equal to $v=0.3$. The Young's modulus of the interface is denoted by $E_{i}$. The analyses are carried out under plane strain hypothesis, considering different geometrical (thickness) and mechanical (stiffness) properties of the interface.

The influence of two parameters

- the relative thickness of the interphase $\frac{\varepsilon}{L}$;

- the relative stiffness of the interphase $\frac{E_{i}}{E_{a}}$,

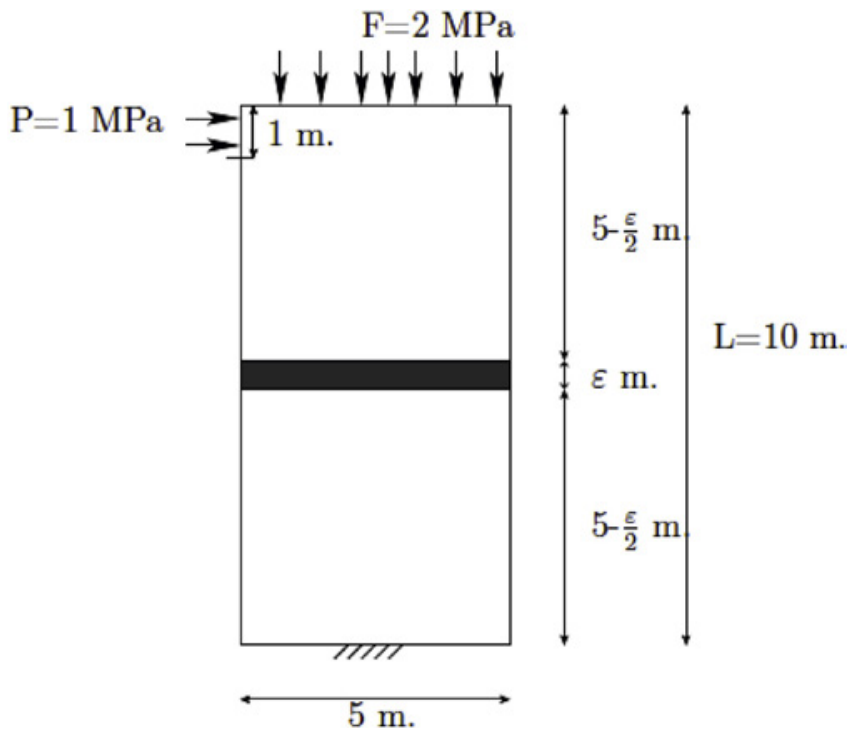

Fig. 1. Geometry of the problem ( $\varepsilon=0$ for the interface problem). is investigated in order to evaluate the accuracy of the various modeling proposed in the previous sections. In particular, the quality of the solutions is evaluated considering the $L_{2}$-relative error:

$e=\frac{\left\|u^{\varepsilon}-u_{\text {model }}^{\text {order }}\right\|}{\left\|u^{\varepsilon}\right\|}$

where $u^{\varepsilon}$ is the reference solution computed using the three-phases problem with a fine finite element mesh, and $u_{\text {model }}^{\text {order }}$ indicates the different interface model solutions, with model = soft or hard and order $=0$ or 1 .

In particular, the relative errors for the following models are reported:

- soft interface at order 0 (Table 1);

- hard interface at order 0 (Table 2);

- soft interface at order 1 (Table 3);

- hard interface at order 1 (Table 4);

- hard interface at order 1 using semi-implicit formulation (64) (Table 5).

It can observed that, in all the cases herein considered, the soft interface modeling, both at order 0 and 1 (see Tables 1 and 3), is able to provide an acceptable solution, with a relative error less than $10 \%$.

If the relative thickness of the interface is less than $1 \%$, then the relative error is also less than $1 \%$, except in the case of a relative stiffness between the adhesive and the adherents equal to 10 (representing a very hard adhesive).

In Table 2, it can be highlighted that the hard interface modeling at order 0 is not suitable when the interface is thick or when its relative stiffness is small. In that cases, the hard interface modeling at order 1 (see Table 4) significantly improves the results, except if the interface has both a large thickness and a small stiffness.

Table 5 presents the results obtained considering the semi-implicit formulation (64) of the hard interface at order 1 . This formulation provides generally satisfactory results, excepted in the case of a very stiff and large thickness interface. Except in the latter case, the results numerically confirms that the semi-implicit formulation (64) is equivalent to the soft interface modeling at order 1 .

Fig. 2 presents the plot of the convergence to zero of the relative error versus the thickness of the glue computed for the various models, in the case of the relative stiffness of the glue equal to $0.1 \%$ (second lines of the tables), that can be considered as a soft interface (small relative stiffness). The figure confirms and clarifies the remarks above described from the analysis of the results reported in the tables.

As expected, the hard interface modeling both at order 0 and 1 converge slowly, whereas the soft interface modeling at order 0 and 1 , and the semi-implicit modeling provide close results and converge faster. This demonstrates that, in this case, the soft interface modeling is able to correctly approximate the solution $u^{\varepsilon}$, and the implicit formulation for hard interface is equivalent to the soft one.

In Fig. 3, the convergence of the relative error is presented for a stiffer interface, setting $E_{i} / E_{a}=2$. In this case, since the interface can be considered as rigid, the plot shows that the soft interface modeling both at order 0 and 1 converge slowly, whereas the hard interface modeling at order 0 and 1 converge faster. It can be noticed that the semi-implicit formulation provides good results, equivalent to the explicit hard one at order 1 in this case.

\subsection{Single lap joint}

The response of a structural system consisting in two beams joined by a single lap bond is studied; the structural scheme is illustrated in Fig. 4. The upper beam is clamped on the left, while the lower beam is submitted to a distributed horizontal load $F=2 \mathrm{MPa}$ on the right.

The structure is characterized by the following geometrical and 
Table 1

Relative difference $\left\|u^{\varepsilon}-u_{\text {soft }}^{0}\right\| /\left\|u^{\varepsilon}\right\|$ at order 0 (red: large relative error; green: small relative error; black: acceptable relative error).

\begin{tabular}{|c|c|c|c|c|c|c|c|}
\hline & 0.0001 & 0.0005 & 0.001 & 0.005 & 0.01 & 0.05 & 0.1 \\
\hline $10^{-4}$ & $1.389 \cdot 10^{-4}$ & $2.303 \cdot 10^{-4}$ & $6.449 \cdot 10^{-4}$ & $4.490 \cdot 10^{-3}$ & $8.077 \cdot 10^{-3}$ & $4.286 \cdot 10^{-2}$ & $8.581 \cdot 10^{-2}$ \\
\hline $10^{-3}$ & $2.478 \cdot 10^{-4}$ & $2.415 \cdot 10^{-4}$ & $1.434 \cdot 10^{-4}$ & $3.064 \cdot 10^{-3}$ & $6.614 \cdot 10^{-3}$ & $4.127 \cdot 10^{-2}$ & $8.433 \cdot 10^{-2}$ \\
\hline $10^{-2}$ & $2.691 \cdot 10^{-4}$ & $4.510 \cdot 10^{-4}$ & $6.696 \cdot 10^{-4}$ & $9.984 \cdot 10^{-4}$ & $1.014 \cdot 10^{-3}$ & $2.863 \cdot 10^{-2}$ & $7.111 \cdot 10^{-2}$ \\
\hline $10^{-1}$ & $2.723 \cdot 10^{-4}$ & $4.902 \cdot 10^{-4}$ & $7.969 \cdot 10^{-4}$ & $2.822 \cdot 10^{-3}$ & $5.002 \cdot 10^{-3}$ & $1.042 \cdot 10^{-2}$ & $2.087 \cdot 10^{-2}$ \\
\hline 1 & $2.809 \cdot 10^{-4}$ & $5.411 \cdot 10^{-4}$ & $9.082 \cdot 10^{-4}$ & $3.655 \cdot 10^{-3}$ & $7.179 \cdot 10^{-3}$ & $3.451 \cdot 10^{-2}$ & $6.832 \cdot 10^{-2}$ \\
\hline 10 & $3.714 \cdot 10^{-4}$ & $1.019 \cdot 10^{-3}$ & $1.823 \cdot 10^{-3}$ & $7.030 \cdot 10^{-3}$ & $1.251 \cdot 10^{-2}$ & $4.900 \cdot 10^{-2}$ & $9.493 \cdot 10^{-2}$ \\
\hline
\end{tabular}

Table 2

Relative difference $\left\|u^{\varepsilon}-u_{\text {hard }}^{0}\right\| /\left\|u^{\varepsilon}\right\|$ at order 0 (red: large relative error; green: small relative error; black: acceptable relative error).

\begin{tabular}{r|c|c|c|c|c|c|c}
\hline${\frac{E_{i}}{E_{a}}}^{\frac{\varepsilon}{L}}$ & 0.0001 & 0.0005 & 0.001 & 0.005 & 0.01 & 0.05 & 0.1 \\
\hline $10^{-4}$ & $4.049 \cdot 10^{-1}$ & $7.757 \cdot 10^{-1}$ & $8.743 \cdot 10^{-1}$ & $9.722 \cdot 10^{-1}$ & $9.860 \cdot 10^{-1}$ & $9.972 \cdot 10^{-1}$ & $9.986 \cdot 10^{-1}$ \\
$10^{-3}$ & $6.376 \cdot 10^{-2}$ & $2.530 \cdot 10^{-1}$ & $4.049 \cdot 10^{-1}$ & $7.763 \cdot 10^{-1}$ & $8.750 \cdot 10^{-1}$ & $9.732 \cdot 10^{-1}$ & $9.868 \cdot 10^{-1}$ \\
$10^{-2}$ & $6.650 \cdot 10^{-3}$ & $3.281 \cdot 10^{-2}$ & $6.335 \cdot 10^{-2}$ & $2.525 \cdot 10^{-1}$ & $4.050 \cdot 10^{-1}$ & $7.817 \cdot 10^{-1}$ & $8.811 \cdot 10^{-1}$ \\
$10^{-1}$ & $4.693 \cdot 10^{-4}$ & $2.991 \cdot 10^{-3}$ & $6.112 \cdot 10^{-3}$ & $3.052 \cdot 10^{-2}$ & $5.931 \cdot 10^{-2}$ & $2.459 \cdot 10^{-1}$ & $4.030 \cdot 10^{-1}$ \\
1 & $2.204 \cdot 10^{-4}$ & $2.160 \cdot 10^{-4}$ & $2.434 \cdot 10^{-4}$ & $2.178 \cdot 10^{-4}$ & $2.923 \cdot 10^{-4}$ & $2.227 \cdot 10^{-4}$ & $2.219 \cdot 10^{-4}$ \\
10 & $3.648 \cdot 10^{-4}$ & $9.865 \cdot 10^{-4}$ & $1.756 \cdot 10^{-3}$ & $6.701 \cdot 10^{-3}$ & $1.184 \cdot 10^{-2}$ & $4.546 \cdot 10^{-2}$ & $8.752 \cdot 10^{-2}$ \\
\hline
\end{tabular}

Table 3

Relative difference $\left\|u^{\varepsilon}-u_{\text {soft }}^{0}-\varepsilon u_{\text {soft }}^{1}\right\| /\left\|u^{\varepsilon}\right\|$ at order 1 (red: large relative error; green: small relative error; black: acceptable relative error).

\begin{tabular}{r|c|c|c|c|c|c|c}
\hline${\frac{E_{i}}{E_{a}}}^{\frac{\varepsilon}{L}}$ & 0.0001 & 0.0005 & 0.001 & 0.005 & 0.01 & 0.05 & 0.1 \\
\hline $10^{-4}$ & $1.597 \cdot 10^{-4}$ & $1.583 \cdot 10^{-4}$ & $5.245 \cdot 10^{-4}$ & $4.026 \cdot 10^{-3}$ & $7.189 \cdot 10^{-3}$ & $3.809 \cdot 10^{-2}$ & $7.895 \cdot 10^{-2}$ \\
$10^{-3}$ & $2.654 \cdot 10^{-4}$ & $3.544 \cdot 10^{-4}$ & $3.239 \cdot 10^{-4}$ & $2.370 \cdot 10^{-3}$ & $5.494 \cdot 10^{-3}$ & $2.597 \cdot 10^{-2}$ & $7.784 \cdot 10^{-2}$ \\
$10^{-2}$ & $2.800 \cdot 10^{-4}$ & $5.277 \cdot 10^{-4}$ & $8.493 \cdot 10^{-4}$ & $1.950 \cdot 10^{-3}$ & $1.663 \cdot 10^{-3}$ & $8.694 \cdot 10^{-3}$ & $6.794 \cdot 10^{-2}$ \\
$10^{-1}$ & $2.818 \cdot 10^{-4}$ & $5.449 \cdot 10^{-4}$ & $9.118 \cdot 10^{-4}$ & $3.451 \cdot 10^{-3}$ & $6.223 \cdot 10^{-3}$ & $2.516 \cdot 10^{-2}$ & $2.336 \cdot 10^{-2}$ \\
1 & $2.904 \cdot 10^{-4}$ & $5.938 \cdot 10^{-4}$ & $1.013 \cdot 10^{-3}$ & $4.092 \cdot 10^{-3}$ & $7.778 \cdot 10^{-3}$ & $3.726 \cdot 10^{-2}$ & $2.204 \cdot 10^{-2}$ \\
10 & $3.817 \cdot 10^{-4}$ & $5.938 \cdot 10^{-4}$ & $1.936 \cdot 10^{-3}$ & $7.477 \cdot 10^{-3}$ & $1.307 \cdot 10^{-2}$ & $3.878 \cdot 10^{-2}$ & $3.790 \cdot 10^{-2}$ \\
\hline
\end{tabular}

Table 4

Relative difference $\left\|u^{\varepsilon}-u_{\text {hard }}^{0}-\varepsilon u_{\text {hard }}^{1}\right\| /\left\|u^{\varepsilon}\right\|$ at order 1 (red: large relative error; green: small relative error; black: acceptable relative error).

\begin{tabular}{|c|c|c|c|c|c|c|c|}
\hline & 0.0001 & 0.0005 & 0.001 & 0.005 & 0.01 & 0.05 & 0.1 \\
\hline $10^{-4}$ & $3.029 \cdot 10^{-2}$ & $5.567 \cdot 10^{-2}$ & $6.265 \cdot 10^{-2}$ & $7.242 \cdot 10^{-2}$ & $7.636 \cdot 10^{-2}$ & $1.073 \cdot 10^{-1}$ & $1.434 \cdot 10^{-1}$ \\
\hline $10^{-3}$ & $5.868 \cdot 10^{-3}$ & $1.990 \cdot 10^{-2}$ & $3.055 \cdot 10^{-2}$ & $5.851 \cdot 10^{-2}$ & $6.819 \cdot 10^{-2}$ & $1.049 \cdot 10^{-1}$ & $1.418 \cdot 10^{-1}$ \\
\hline $10^{-2}$ & $5.593 \cdot 10^{-4}$ & $3.173 \cdot 10^{-3}$ & $5.848 \cdot 10^{-3}$ & $2.085 \cdot 10^{-2}$ & $3.330 \cdot 10^{-2}$ & $8.555 \cdot 10^{-2}$ & $1.275 \cdot 10^{-1}$ \\
\hline $10^{-1}$ & $1.560 \cdot 10^{-4}$ & $1.622 \cdot 10^{-4}$ & $4.673 \cdot 10^{-4}$ & $3.004 \cdot 10^{-3}$ & $5.820 \cdot 10^{-3}$ & $2.942 \cdot 10^{-2}$ & $6.059 \cdot 10^{-2}$ \\
\hline 1 & $2.216 \cdot 10^{-4}$ & $2.221 \cdot 10^{-4}$ & $2.555 \cdot 10^{-4}$ & $2.788 \cdot 10^{-4}$ & $4.147 \cdot 10^{-4}$ & $8.396 \cdot 10^{-4}$ & $1.459 \cdot 10^{-3}$ \\
\hline 10 & $2.236 \cdot 10^{-4}$ & $2.036 \cdot 10^{-4}$ & $1.877 \cdot 10^{-4}$ & $1.813 \cdot 10^{-3}$ & $5.260 \cdot 10^{-3}$ & $4.347 \cdot 10^{-2}$ & $9.851 \cdot 10^{-2}$ \\
\hline
\end{tabular}

Table 5

Relative difference $\left\|u^{\varepsilon}-\widetilde{u}_{\text {hard }}^{0}-\varepsilon \widetilde{u}_{\text {hard }}^{1}\right\| / u^{\varepsilon}$ at order 1 , using the semi-implicit formulation (red: large relative error; green: small relative error; black: acceptable relative error).

\begin{tabular}{|c|c|c|c|c|c|c|c|}
\hline & 0.0001 & 0.0005 & 0.001 & 0.005 & 0.01 & 0.05 & 0.1 \\
\hline $10^{-4}$ & $1.125 \cdot 10^{-4}$ & $2.880 \cdot 10^{-4}$ & $7.085 \cdot 10^{-4}$ & $4.541 \cdot 10^{-3}$ & $8.104 \cdot 10^{-3}$ & $4.269 \cdot 10^{-2}$ & $8.546 \cdot 10^{-2}$ \\
\hline $10^{-3}$ & $2.028 \cdot 10^{-4}$ & $8.602 \cdot 10^{-5}$ & $2.506 \cdot 10^{-4}$ & $3.645 \cdot 10^{-3}$ & $7.247 \cdot 10^{-3}$ & $4.176 \cdot 10^{-2}$ & $8.452 \cdot 10^{-2}$ \\
\hline $10^{-2}$ & $2.199 \cdot 10^{-4}$ & $1.993 \cdot 10^{-4}$ & $1.805 \cdot 10^{-4}$ & $1.189 \cdot 10^{-3}$ & $3.539 \cdot 10^{-3}$ & $3.432 \cdot 10^{-2}$ & $7.610 \cdot 10^{-2}$ \\
\hline $10^{-1}$ & $2.209 \cdot 10^{-4}$ & $2.197 \cdot 10^{-4}$ & $2.480 \cdot 10^{-4}$ & $1.391 \cdot 10^{-4}$ & $4.473 \cdot 10^{-4}$ & $1.194 \cdot 10^{-2}$ & $3.591 \cdot 10^{-2}$ \\
\hline 1 & $2.086 \cdot 10^{-4}$ & $2.369 \cdot 10^{-4}$ & $2.717 \cdot 10^{-4}$ & $2.744 \cdot 10^{-4}$ & $4.026 \cdot 10^{-4}$ & $8.274 \cdot 10^{-4}$ & $1.432 \cdot 10^{-3}$ \\
\hline 10 & $2.254 \cdot 10^{-3}$ & $4.350 \cdot 10^{-4}$ & $1.230 \cdot 10^{-3}$ & $2.158 \cdot 10^{-3}$ & $5.803 \cdot 10^{-3}$ & $4.658 \cdot 10^{-2}$ & $1.047 \cdot 10^{-1}$ \\
\hline
\end{tabular}




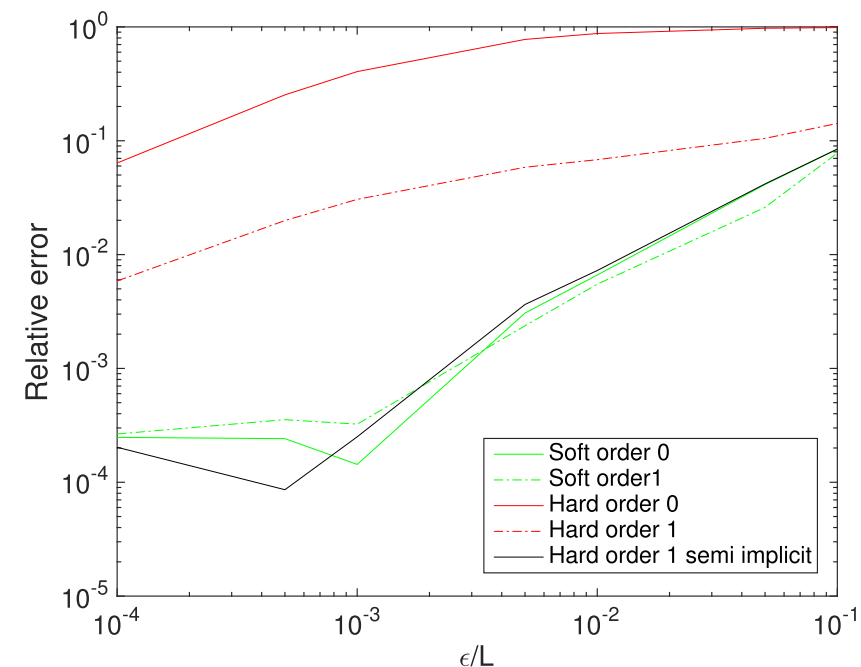

Fig. 2. Convergence results for $\frac{E_{i}}{E_{a}}=10^{-3}$.

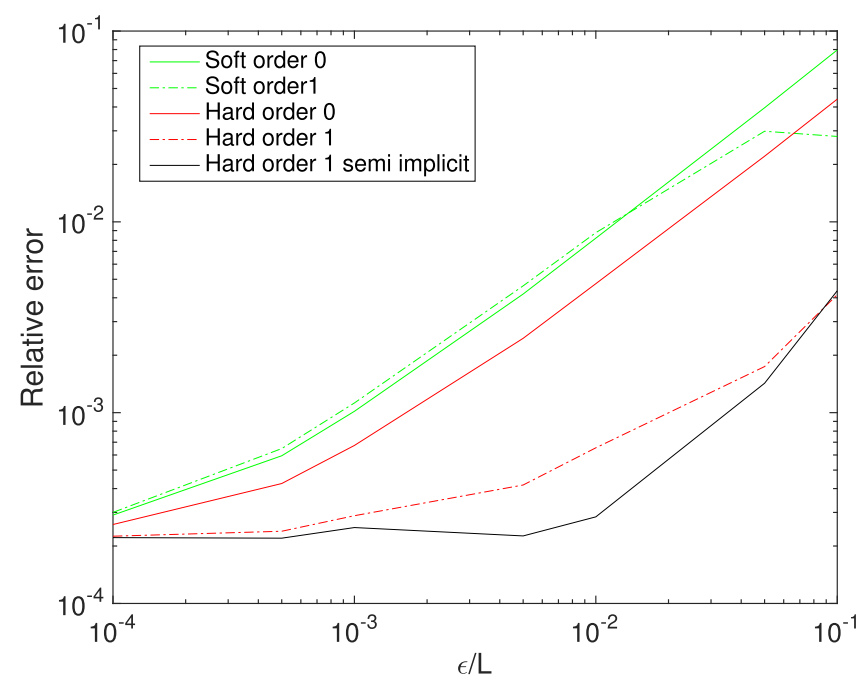

Fig. 3. Convergence results for $\frac{E_{i}}{E_{a}}=2$.

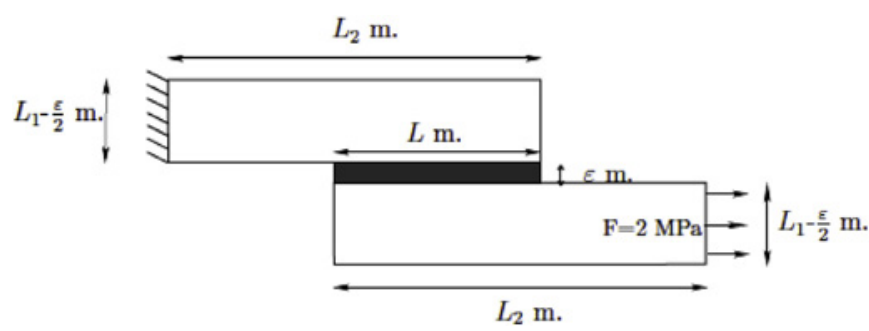

Fig. 4. Geometry of the problem ( $\varepsilon=0$ for the interface problem).

mechanical parameters:

$L_{2}=10, \quad L=1, \quad \varepsilon=0.01$

where, as in the previous application, all the lengths are measured in meters. Note that $L$ represents the length of the glued zone. The bonded beams system is modeled considering a two-dimensional plane strain assumption, so that each beam has a width equal to $1 \mathrm{~m}$.

Different values for the beam height $L_{1}$ and for the elastic moduli ratio $\frac{E_{i}}{E_{a}}$ are considered in the computations in order to investigate on effectiveness of the hard and soft interface models and on the matching condition between the external and the internal displacement jump.

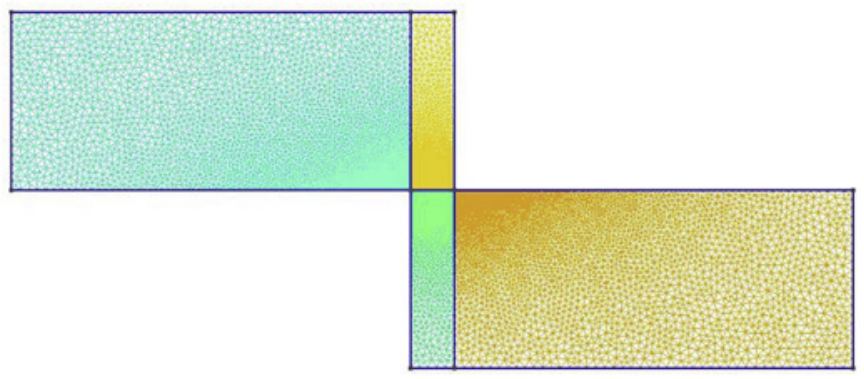

Fig. 5. The beam with $L_{1}=4$.

Three cases are studied:

- thick beams bonded by hard interface

- thin beams bonded by hard interface

- thin beams bonded by soft interface

In the following, the displacement jump computed for the surfaces $S_{+}$and $S_{-}$assumed to belong to the boundary of the two adherents is named as external jump of displacement; analogously, the displacement jump computed for the surfaces $S_{+}$and $S_{-}$assumed to belong to the boundary of the adhesive is named as internal jump of displacement. The matching conditions (12), (13) and (14) enforce that the external jump is equal to internal jump.

In the following, the internal jump of the displacement $u_{1}$, evaluated as:

$\left[\left[u_{1}\right]\right]\left(x_{1}, x_{2}=0\right)=u_{1}\left(x_{1}, 0^{+}\right)-u_{1}\left(x_{2}, 0^{-}\right)$,

and the external jump of the displacement $u_{1}$, evaluated as:

$\left[u_{1}\right]_{e}\left(x_{1}, x_{2}=0\right)=u_{1}\left(x_{1},+\frac{\varepsilon}{2}\right)-u_{1}\left(x_{1},-\frac{\varepsilon}{2}\right)$,

are computed for:

1. the three phases computation;

2. the hard interface modeling at order 0 ;

3 . the solution obtained at order 1 , prescribing the relative displacement equal to:

$\varepsilon \frac{2(1+v)}{E} \sigma_{12}^{0}\left(x_{1}, 0\right)-u_{2,1}^{0}\left(x_{1}, 0\right)-u_{1,2}^{0}\left(x_{1}, 0\right) ;$

4. the hard interface modeling at order 1 , computed considering the

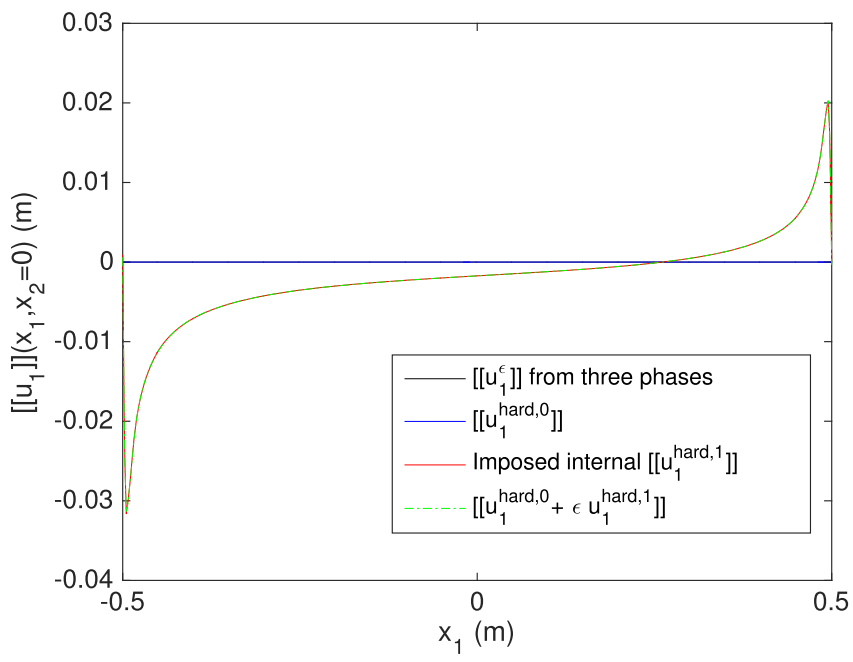

Fig. 6. Internal jumps in the displacement $u_{1}$. 


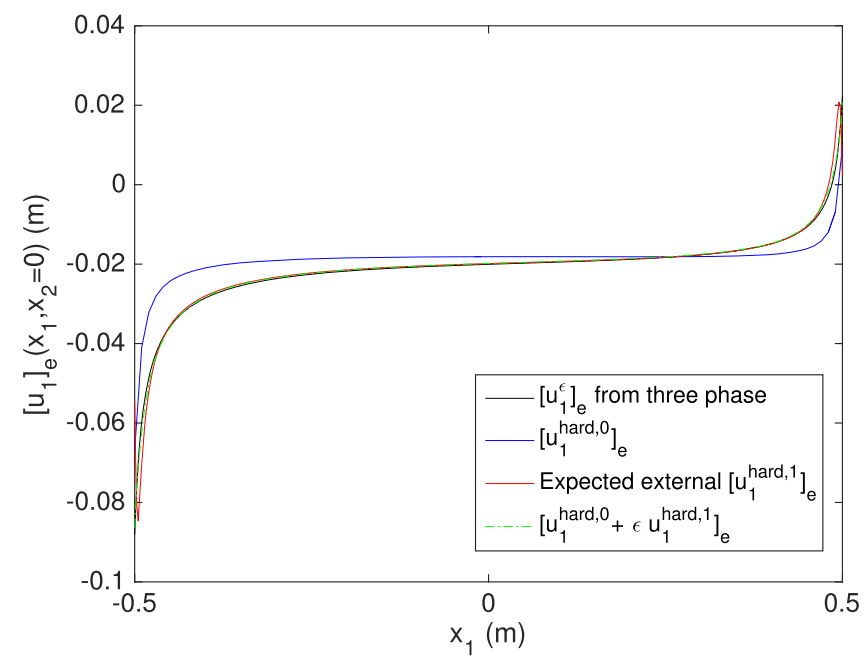

Fig. 7. External jumps in the displacement $u_{1}$.

solution provided from the prescribed jump introduced above.

\subsubsection{Thick beams bonded by hard interface}

Initially, the case of thick beams is investigated. The thickness of the two beams is set as $L_{1}=4$, while the elastic moduli ratio is taken as $E_{i} / E_{a}=1 / 2$, corresponding to a hard interface. In Fig. 5 the geometry of the problem, with the very fine mesh adopted for the computations, is illustrated.

It can be remarked that in the second case, the displacement jump has to be equal to zero, as perfect material continuity is ensured for the two adherents; indeed, in this case, the adhesive phase is reduced to zero.

In Fig. 6, the internal jumps of the displacement $u_{1}$, evaluated from formula (68), for the four considered cases are plotted. It results that the curve of the second case is equal to zero, while the three remaining ones overlap each other, showing that the numerical computation is able to take into account the prescribed value. Thus, the expected results are recovered from the computations.

Analogously, the external jumps of the displacement $u_{1}$, evaluated from formula (69), are presented in Fig. 7 for the same cases investigated above.

In this case, one can observe that curves obtained from assumptions corresponding to points 1,3 and 4 overlaps each other. Thus, the prediction of the external jump (curve 3 ) and the observed one (curve 4) correspond to that of the three phase computations (curve 1). So the modeling and the matching are correct.

\subsubsection{Thin beams bonded by hard interface}

In this section, the same computations above described are performed considering the case of two thin bonded beams. In particular, it is set $L_{1}=4$. The structural scheme with the mesh adopted for the computation is illustrated in Fig. 8. Computations are performed assuming the elastic moduli ratio is taken as $E_{i} / E_{a}=1 / 2$, corresponding to a hard interface.

In Fig. 9, the internal jump of the displacement $u_{1}$ given by formula (68) is plotted for the different solutions.

As for the case of thick beams, it can be observed that the curve corresponding to the second case is everywhere equal to zero, and the

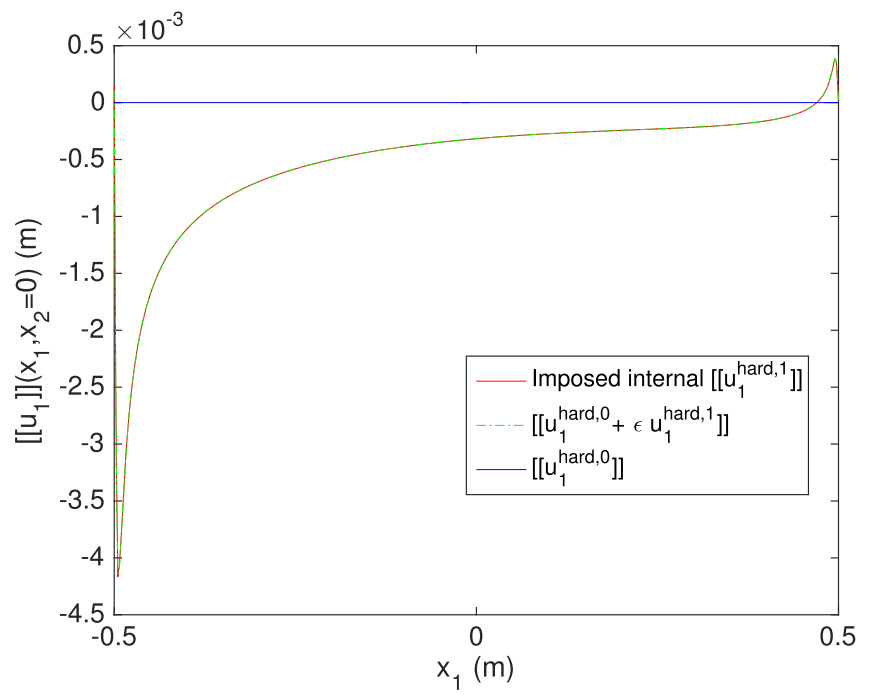

Fig. 9. Internal jumps in the displacement $u_{1}$.

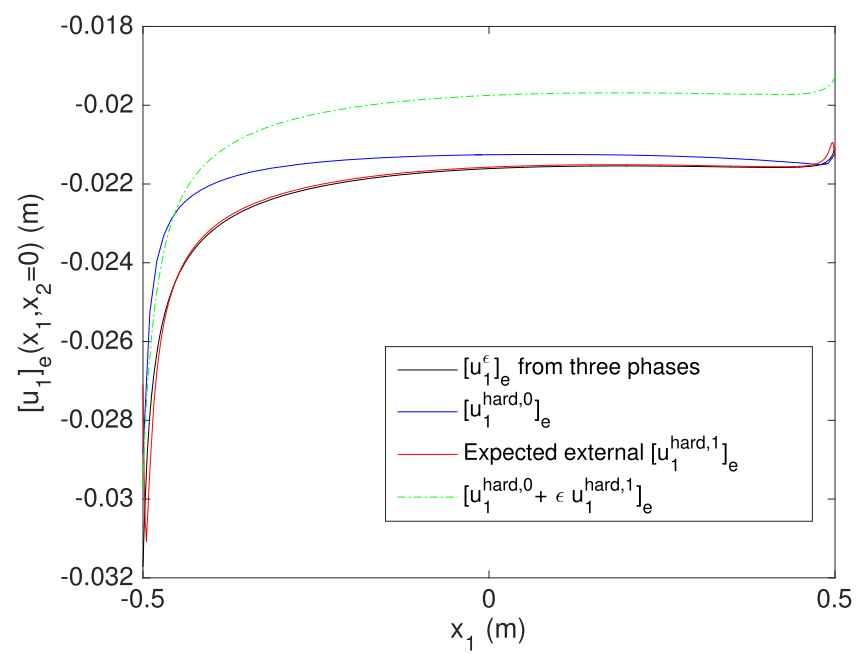

Fig. 10. External jumps in the displacement $u_{1}$.

two last ones overlap each other, showing that also for thin beams with hard interface, the numerical computation is able to take into account the prescribed value.

Fig. 10 provides the external jump of the displacement $u_{1}$ determined by formula (69). In this case, it is observed that only curves 1 and 3 overlap each other, showing that the prediction of the external jump (curve 3 , before the matching) is the good one and correspond to that of the three phases computation (curve 1), but the observed one (curve 4), computed as internal jump with matching, is not the correct one when the beam is thin.

If the matching is not enforced, i.e. if the jumps between the two adherents (with no materials between them) is considered directly, one can observe in Fig. 11 that curves 1, 3 and 4 overlaps each other. This means that for thin beams, the computation without internal jump with matching leads to satisfactory results. Moreover, it is noticed that in this

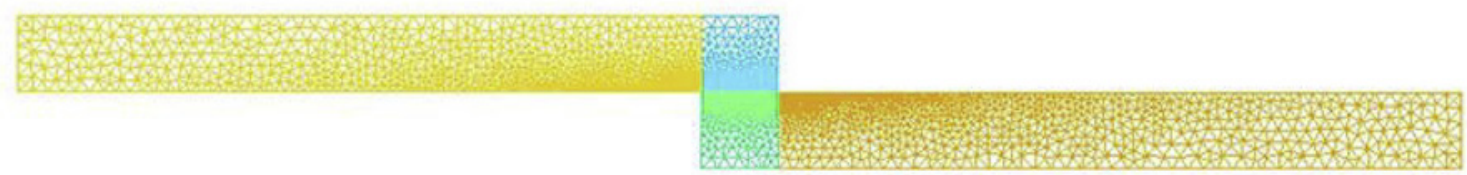

Fig. 8. The beam with $L_{1}=1$. 


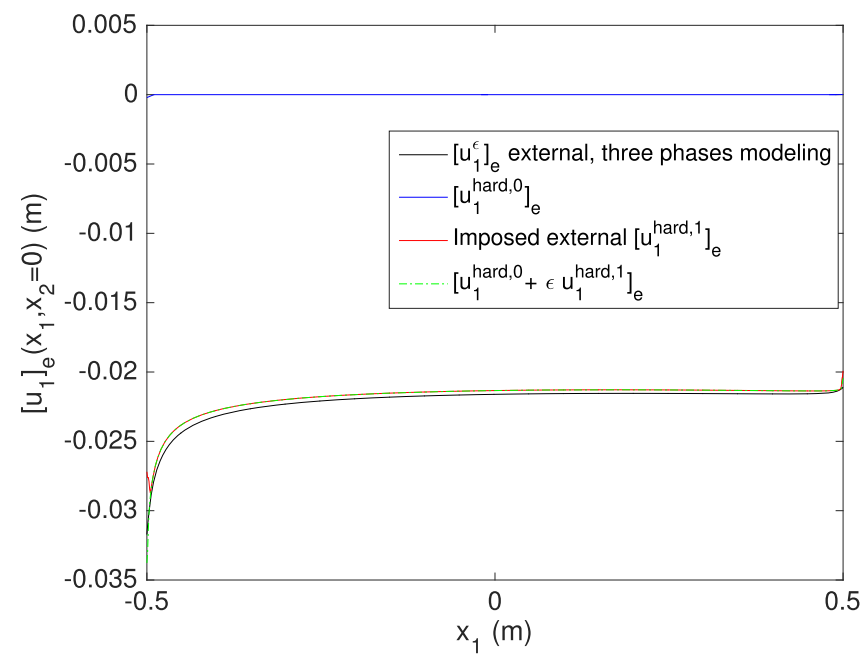

Fig. 11. External jumps in the displacement $u_{1}$.

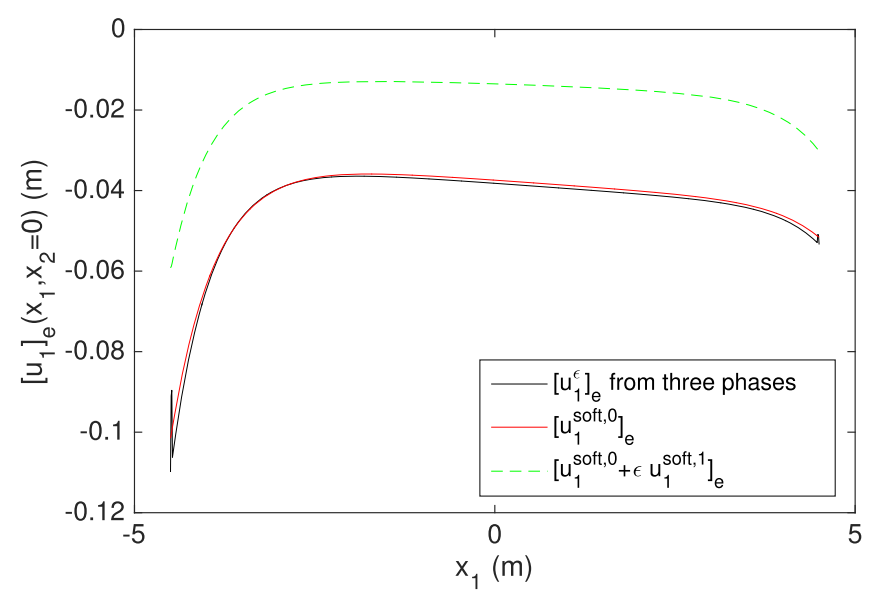

Fig. 12. External jumps in the displacement $u_{1}$ with soft interface and matching.

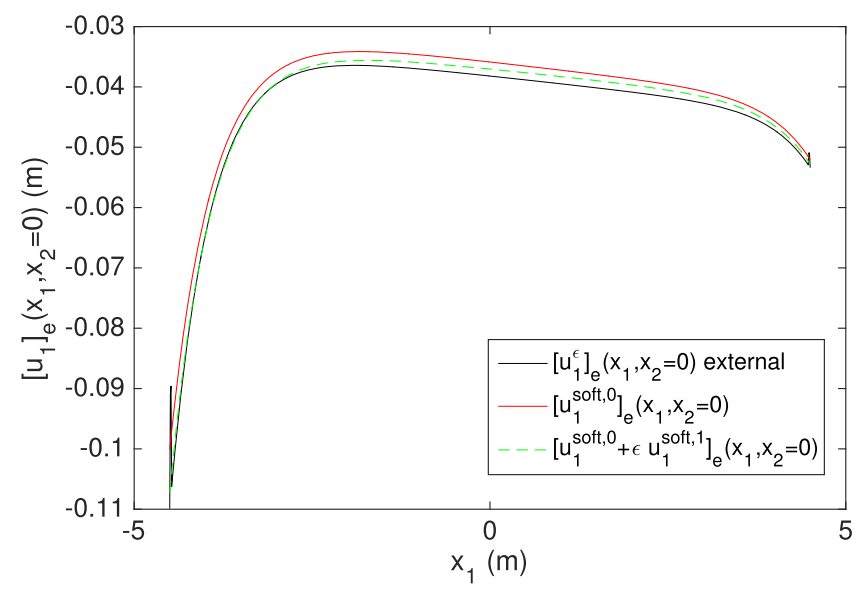

Fig. 13. External jumps in the displacement $u_{1}$ with soft interface and no matching.

case the solution on the whole structure is better than in the other simulations.

\subsubsection{Thin beams bonded by soft interface}

Finally, the case of soft interface, obtained setting the moduli ratio $E_{i} / E_{a}=0.001$ for very thin beams $\left(L_{2}=0.1\right)$ is considered.
In that case, the jump in the displacements for the first component is not correctly approximated, due to the matching (see Fig. 12). If the matching is not considered, the results is improved (see Fig. 13). It is noticed that for soft interface, unsatisfactory results are obtained for thinner beams than in the case of hard interface.

\section{Conclusion}

In this paper, mathematical and numerical methods based on asymptotic expansions are proposed for the modeling of bonded interfaces. A linear elastic response of the adhesive and adherents is considered, in the frame of small strains and small displacements theory. Two different types of material adhesive are considered: the case of a hard material, whose elasticity coefficients are independent of the adhesive thickness, $\varepsilon$, and the case of a soft material, whose elasticity coefficients rescale like $\varepsilon$.

In the first part of the paper, some recent results of asymptotic analysis for soft or stiff interfaces are reviewed [9,28,29]. For the two cases of material behavior, the asymptotic expansions of the displacement and stress vector fields are introduced and the effect of higher order terms is taken into account. Using the classical scheme of matched asymptotic expansions, the interface conditions are obtained, linking the stress vector field and its jump to the displacement vector field and its jump at the interface, which is the geometric limit of the adhesive layer as its thickness parameter $\varepsilon$ goes to zero. Following a Remark already introduced in Ref. [28], an implicit interface condition is presented, able to account for the interface conditions for both the two considered cases of hard and soft interfaces at the orders zero and one.

Taking into account higher order terms of the asymptotic expansions, this implicit form of interface condition is expected to provide an improved approximation of the behavior of the thin adhesive interface when compared to the classical spring-type interface law or to the case of perfect contact between the adherents. To verify this conjecture, some numerical examples are analyzed in the second part of the paper. The numerical results show that

- when the thickness of the adhesive is much smaller than a characteristic length of the adherents,

- for adhesives with stiffness much lower than that of the adherents, the soft interface model shows a small relative error with respect to the exact solution, i.e. the numerical solution for the three layers;

- for adhesives with stiffness comparable to that of the adherents, the hard interface model shows a small relative error with respect to the exact solution;

- in both cases, the interface models at order one converges better than the interface models at order zero;

- the semi-implicit model is always a better approximation;

- when the thickness of the adhesive becomes comparable with a characteristic length of the adherents,

- the solution obtained using matching conditions calculated for the order one is not a good approximation of the exact solution;

- an improved solution can be obtained if the matching at order one is taken to be the same of the order zero, i.e. the continuity of all fields without additional terms;

- a possible explanation of the failing of the matching at the order one is that a problem with to parameters occurs when the adhesive and adherents have comparable thicknesses. This occurrence has be studied using an adapted asymptotic expansion methods.

The last Remark has to be carefully taken into account when studying the bonding of two beams or layered structures with comparable thicknesses. This case, which has relevant applications in many fields, is addressed in a work in progress. 


\section{Appendix A. Supplementary data}

Supplementary data related to this article can be found at https:// doi.org/10.1016/j.compositesb.2018.08.076.

\section{References}

[1] Alfano G, Sacco E. Combining interface damage and friction in a cohesive-zone model. Int J Numer Meth Eng 2006;68(5):542-82.

[2] Ascione L, Berardi VP, Feo L, Mancusi G. A numerical evaluation of the interlaminar stress state in externally frp plated rc beams. Compos B Eng 2005;36(1):83-90.

[3] Becker Roland, Peter Hansbo, Rolf Stenberg. A finite element method for domain decomposition with non-matching grids. ESAIM Math Model Numer Anal 2003;37(2):209-25.

[4] Carozzi FG, Milani G, Poggi C. Mechanical properties and numerical modeling of fabric reinforced cementitious matrix (frcm) systems for strengthening of masonry structures. Compos Struct 2014;107:711-25.

[5] Ciarlet PG. A justification of a nonlinear model in plate theory. Comput Meth Appl Mech Eng 1979;17:227-58.

[6] Ciarlet PG. Mathematical elasticity: three-dimensional elasticity. Number 1 in mathematical elasticity: three-dimensional elasticity. North-Holland. 1994.

[7] Demarkles LR. Investigation on the use of rubber analogue in the study of the stress distribution in riveted and cemented joint NASA; 1955. Technical Report TN No. 3413.

[8] Dumont S, Goubet O, Ha-Duong T, Villon P. Meshfree methods and boundary conditions. Int J Numer Meth Eng 2006;67:989-1011.

[9] Dumont S, Lebon F, Rizzoni R. An asymptotic approach to the adhesion of thin stiff films. Mech Res Commun 2014;58:24-35.

[10] Triantafillou T, editor. Textile fibre composites in civil engineering. Woodhead Publishing; 2016.

[11] Kim Y, editor. Advanced composites in bridge construction and repair. Woodhead Publishing; 2014.

[12] Erdogan F, Ratwani M. Stress distribution in bonded joints. J Compos Mater 1971;5:378-93.

[13] Fouchal F, Lebon F, Titeux I. Contribution to the modelling of interfaces in masonry construction. Construct Build Mater 2009;23(6):2428-41.

[14] Goland M, Reissner E. Stresses in cemented joints. J Appl Mech 1944;11:A17-27.

[15] Grande E, Imbimbo M, Sacco E. Finite element analysis of masonry panels strengthened with frps. Compos B Eng 2013;45(1):1296-309.

[16] Hollaway LC, Teng JG, editors. Strengthening and rehabilitation of civil infrastructures using fibre-reinforced polymer (FRP) composites. Woodhead Publishing; 2008.
[17] Hollaway LC, Leeming M. Strengthening of reinforced concrete structures Woodhead Publishing; 1999.

[18] Ispir M, Dalgic KD, Ilki A. Hybrid confinement of concrete through use of low and high rupture strain frps. Compos B Eng 2018;153:243-55.

[19] Lignola GP, Caggegi C, Ceroni F, De Santis S, Krajewski P, LourenÃßo PB, Morgant M, Papanicolaou C, Pellegrino C, Prota A, Zuccarino L. Performance assessment of basalt frcm for retrofit applications on masonry. Compos B Eng 2017;128:1-18.

[20] Lotfi HR, Shing PB. Interface model applied to fracture of masonry structures. J Struct Eng 1994;120(1):63-80.

[21] Lourenço PB, Rots JG. Multisurface interface model for analysis of masonry structures. J Eng Mech 1997;123(7):660-8.

[22] Marfia S, Sacco E, Toti J. A coupled interface-body nonlocal damage model for frp strengthening detachment. Comput Mech 2012;50(3):335-51.

[23] Nairn JA. Numerical implementation of imperfect interfaces. Comput Mater Sci 2007;40:525-36.

[24] Nitsche J. Convergence of nonconforming methods. Mathematical aspects of finite elements in partial differential equations, pages 15-53, proc. Sympos., math. Res. Center, University of Wisconsin, Madison. New York: Academic Press; 1974.

[25] Olivito RS, Codispoti R, Cevallos OA. Bond behavior of flax-frcm and pbo-frcm composites applied on clay bricks: experimental and theoretical study. Compos Struct 2016;146:221-31.

[26] Raoof SM, Koutas LN, Bournas DA. Bond between textile-reinforced mortar (trm) and concrete substrates: experimental investigation. Compos B Eng 2016;98:350-61.

[27] Raous M, Cangémi L, Cocu M. A consistent model coupling adhesion, friction, and unilateral contact. Comput Meth Appl Mech Eng 1999;177(3):383-99.

[28] Rizzoni R, Dumont S, Lebon F, Sacco E. Higher order model for soft and hard elastic interfaces. Int J Solid Struct 2014;51(23-24):4137-48.

[29] Rizzoni R, Lebon F. Asymptotic analysis of an adhesive joint with mismatch strain. Eur J Mech Solid 2012;36(1-8).

[30] Sacco E, Lebon F. A damage-friction interface model derived from micromechanical approach. Int J Solid Struct 2012;49(26):3666-80.

[31] Serpieri R, Albarella M, Sacco E. A 3d two-scale multiplane cohesive-zone model for mixed-mode fracture with finite dilation. Comput Meth Appl Mech Eng 2017;313:857-88.

[32] Stenberg R. On some techniques for approximating boundary conditions in the finite element method. J Comput Appl Math 1995;63:139-48.

[33] Tetta ZC, Triantafillou TC, Bournas DA. On the design of shear-strengthened rc members through the use of textile reinforced mortar overlays. Compos B Eng 2018;147:178-96.

[34] Volkersen O. Die niektraftverteil ung in zugbeanspruchten mit konstanten laschenquerschritten. Luftfahrtforschung 1938;15:41-7.

[35] Zhou A, Qin R, Feo L, Penna R, Lau D. Investigation on interfacial defect criticality of frp-bonded concrete beams. Compos B Eng 2017;113:80-90. 